This is an Accepted Manuscript of an article published by Taylor \& Francis in International Journal of Control on 27/7/2016, available online: http://www.tandfonline.com/10.1080/00207179.2016.1209565. http:// authorservices.taylorandfrancis.com/sharing-your-work/ (Publisher Journal website 8/11/2016)

\title{
Identification and Control of Electromechanical Systems using State-Dependent Parameter Estimation
}

Authors:

Corresponding author: Alexandre Janot, ONERA, 2 Avenue Edouard Belin, 31055 Toulouse, France, alexandre.janot@onera.fr

Peter C. Young, Lancaster University, Lancaster, LA1 4YW, United Kingdom; and Australian National University, Canberra, p.young@lancaster.ac.uk

Maxime Gautier, University of Nantes, IRCCYN, UMR 6597, 1 Rue de la Noë, 44321 Nantes, France, and CNRS, UMR 6597, France, maxime.gautier@irccyn.ec-nantes.fr

Abstract:

This paper addresses the important topic of electromechanical systems identification with an application in robotics. The standard IDIM-LS method of identifying models for robotic systems is based on the use of a continuous-time inverse dynamic model whose parameters are identified from experimental data by linear Least Squares estimation. The paper describes a new alternative but related approach that exploits the State-Dependent-Parameter (SDP) method of nonlinear model estimation and compares its performance with that of IDIM-LS. The SDP method is a two-stage identification procedure able to identify the presence and graphical shape of nonlinearities in dynamic system models with a minimum of a priori assumptions. The performance of the SDP method is evaluated on two electromechanical systems: the Electro-Mechanical Positioning System (EMPS) and the second link of the TX40 robot. The experimental results demonstrate how SDP identification helps to avoid over-reliance on prior conceptions about the nature of the nonlinear characteristics and correct any deficiencies in this regard. Finally, a simulation study shows how the resulting SDP model is able to facilitate nonlinear control system design using linear-like design procedures. 


\section{Introduction}

\subsection{Robot identification}

A standard method of identifying models for robotic systems is based on the use of a continuoustime inverse dynamic model and the application of Least Squares (LS) estimation based on experimental data measured while the robot is being used to track trajectories that excite its full range of dynamic behaviour. For this reason, the Inverse Dynamic Identification Model with Least Squares (IDIM-LS) method, as it is called, is applied with the system operating within a closed loop. It has been applied successfully for the identification of the inertial parameters of several prototypes and industrial robots, (Olsen, Swevers, \& Verdonck, 2002; Swevers, Verdonck, \& De Schutter, 2007; Hollerbach, Khalil, \& Gautier, 2008; Calanca et al., 2011; Gautier, Janot, \& Vandanjon, 2013; Janot, Vandanjon, \& Gautier, 2014a; Janot, Vandanjon, \& Gautier, 2014b), amongst others. Good results can be obtained using this approach provided appropriate bandpass filtering of the joint positions is used to calculate low noise estimates of the joint velocities and accelerations.

Other identification methods have been tried: the Total Least-Squares (Xi, 1995); the extended Kalman filter (Gautier \& Poignet, 2001; Kostic et al., 2004); an algorithm based on Linear Matrix Inequality (LMI) tools (Indri et al., 2002); a Maximum Likelihood (ML) approach (Olsen, Swevers, \& Verdonck, 2002); the Closed-Loop Output-Error method (Östring, Gunnarsson, \&Norrlöf, 2003; Gautier, Janot, \& Vandanjon, 2013); the Set Membership Uncertainty method (Ramdani \& Poignet, 2005); a method which estimates the nonlinear effects in the frequency domain (Wernholt \& Gunnarsson, 2008); and an instrumental variable approach that combines the direct and inverse dynamic models (Janot, Vandanjon, \& Gautier, 2014a; Janot, Vandanjon, \& Gautier, 2014b).

Another promising approach that allows for the identification and estimation of nonlinearities in dynamic systems is the State-Dependent-Parameter (SDP) method of nonlinear model estimation considered in the present paper. This SDP methodology is also a tool that has proven useful in a number of practical applications in various different areas of study (see e.g. (Young, 2011) and the prior references therein).

\subsection{The SDP method}

The SDP method is a statistical identification procedure able to identify the presence and graphical shape of nonlinearities in dynamic system models based on experimental sampled data, with a minimum of assumptions about the nature of the nonlinearities. SDP estimation is carried out in two distinct stages (see e.g. (Young, 2005)): the first, a non-parametric identification stage, where the detailed model structure is identified; and the second, a parametric estimation stage, where the (normally constant) parameters that characterize a selected parameterization of this structure are optimized in some appropriate manner.

In the first, non-parametric stage of SDP modelling, the recursive SDP estimation algorithm is an extension of the stochastic approach to time variable parameter (TVP) estimation (e.g. (Young, 1999) and the prior references therein). As in this TVP case, SDP estimation exploits the power of recursive fixed interval smoothing (FIS) estimation to obtain lag-free, smoothed estimates of the parameter variations. However, it differs from TVP estimation in two important respects (for the detailed description, see (Young 2000, 2001; Young et al., 2001)). First, in order to allow for the rapid variation 
that state dependency can induce in the parameters, the data are sorted into some other, normally non-temporal order (e.g. ascending order of magnitude), so that the rate of change of the parameter variations between samples in this sorted data space is much smaller than in the original observation space. Secondly, an iterative "back-fitting" algorithm is used to allow for the possibility of different state dependency in each parameter.

As we see in the later experimental examples, this nonparametric stage results in a plot of each SDP against its associated state variable, so providing a graphical portrayal of the non-linearity and its location within the model. In other words, non-parametric SDP estimation identifies the structure of the non-linear model, preparatory to the second, parametric estimation stage. Here, the nonlinearities are parameterized in some parametrically efficient manner involving parameters that are normally constant and estimated using a suitable optimization approach (see e.g. (Beven et al., 2012)). It is this two-stage approach that most distinguishes the SDP method from other related approaches to nonlinear system modelling, such as linear and nonlinear parameter varying (LPV/NLPV) methods (e.g. Previdi and Lovera (2003)). The two stages are useful in practice because they help to ensure that the model is parsimonious, with nonlinearities identified and estimated only where they occur within the non-linear SDP model structure.

SDP modelling was developed in this two-stage manner so that it could act as a major tool in DataBased Mechanistic (DBM) modelling (see, e.g. (Young, 1998b) and the prior references therein), where the non-parametric stage often allows for the interpretation of the nonlinear model elements in some physically meaningful manner. Such an interpretation is less straightforward in the case of "black-box" nonlinear models, such as LPV and NLPV, that exploit linear combinations of basis functions or neural net algorithms (see e.g. (Previdi \& Lovera, 2004) and the comment on this in (Young, 2005)). Moreover, it is important to note that the non-parametric model can be used in its own right, depending on the nature of the application, and so it is not always parameterized; whereas parameterization is the norm in LPV identification.

\subsection{Contributions of the paper}

Surprisingly, the SDP method has not received much attention in the field of mechanical engineering (e.g. robotics), although its potential for use in this context was reported some years ago (Young, 1996, 1998). This may due to the fact that the dynamic models of electromechanical systems are most often formulated directly from the Newton's laws or Lagrange's equations. The models are thus available directly in a physically meaningful form and black-box identification and estimation is not considered necessary, although this does mean that the modeller is assuming that the physical interpretation is completely correct. In order to evaluate the performance of the SDP method, it is applied on two electromechanical systems: the Electro-Mechanical Positioning System (EMPS) and the second link of the TX40 robot; and its performance is compared with that of the IDIM-LS method.

The contribution of the paper is four-fold. First, a SDP-based identification method that combines the continuous-time IDM and the SDP method is introduced and experimentally validated on both the EMPS and the second link of the TX40 robot. Second, it is shown how this SDP-based method is able to improve on the performance of the standard IDIM-LS method. Third, a new, iterative SDP-based algorithm is proposed that is able to provide a graphical portrayal of a multi-SDP nonlinearity on the second link of the TX40 robot. It is shown that this iterative SDP-based algorithm yields accurate 
graphical results provided the effects encompassed in the multi-SDP disturbance are sufficiently separable. Finally, a simulation example illustrates how the EMPS model with a SDP identified nonlinearity can be used in the design of a closed loop servomechanism control system.

The rest of the paper is organized as follows. Section 2 reviews the usual LS-based identification method, IDIM-LS, and presents the results obtained by applying this and the new SDP method to data obtained from experiments on the EMPS prototype. Section 3 presents the iterative SDP-based algorithm that is able to extract the non-linearities encompassed in a multi-SDP model and demonstrates its practical utility by application to the second link of the TX40 robot. This is followed by Section 4 that deals with SDP control system design. Concluding remarks are given in the last Section 5.

\section{First case study: the Electro-Mechanical Positioning System}

\subsection{Experimental setup}

The EMPS is a high-precision Electro-Mechanical Positioning System (see Fig. 1). It is a standard configuration of a drive system for the prismatic joints of robots or machine tools. It is connected to a dSPACE digital control system for easy control and data acquisition using Matlab and Simulink software. Its main components are

- A Maxon DC motor equipped with an incremental encoder. As we will see later, the DC motor is position-controlled.

- A Star high-precision low-friction ball screw drive positioning unit and a load in translation.

- An encoder at the extremity of the ball screw. This encoder is not used in this study.

- An accelerometer on the load which measures its acceleration. The accelerometer is not used in this study.

All variables and parameters are given in SI units on the load side.

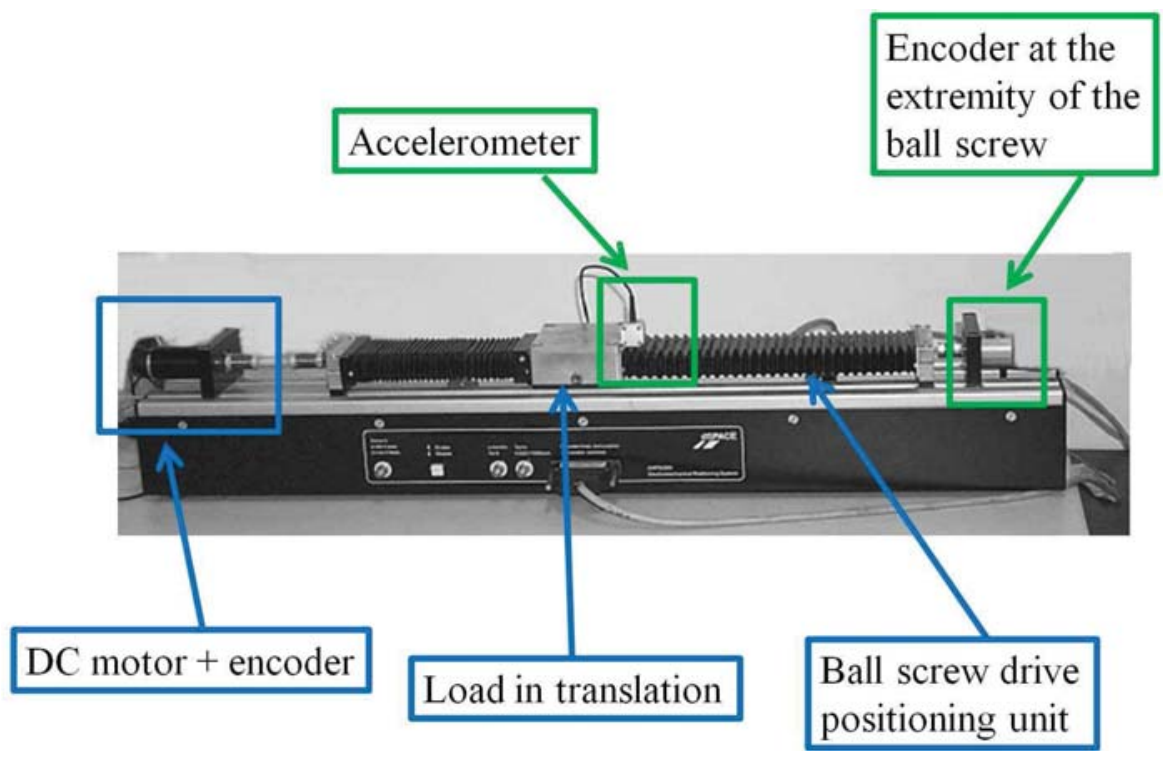

Fig.1. EMPS prototype and its instrumentation 


\subsection{Standard Physically-Based Modelling of the EMPS}

\subsubsection{Direct dynamic model}

The direct dynamic model (DDM) of a robot expresses the acceleration vector as a function of the motor torque, joint position and velocity vector (Khalil \& Dombre, 2002). From Newton's laws, we have

$M \ddot{q}=\tau_{\text {idm }}-\tau_{\text {fric }}-$ offset.

where $q, \dot{q}, \ddot{q}$ are the joint position, velocity and acceleration in $\mathrm{m}, \mathrm{m} \cdot \mathrm{s}^{-1}$ and $\mathrm{m} \cdot \mathrm{s}^{-2}$ respectively; $\tau_{i d m}$ is the motor force in $\mathrm{N}$; $\tau_{\text {fric }}$ is the friction force in $\mathrm{N} ; M$ is the mass in Kg; offset is the offset of measurements. In the case of a 'linear' friction model, $\tau_{\text {fric }}$ is given by

$\tau_{\text {fric }}=F_{v} \dot{q}+F_{c} \operatorname{sign}(\dot{q})$,

where $F_{v}$ and $F_{c}$ are the viscous and Coulomb friction parameters in $\mathrm{N} / \mathrm{m} \cdot \mathrm{s}^{-1}$ and $\mathrm{N}$, respectively.

Although the friction model is usually non-linear (especially at low velocities), this simple friction model is always valid over a range of velocities (Khalil \& Dombre, 2002) and the physical parameters $M, F_{v}, F_{c}$ and offset are referred to as the "dynamic parameters".

\subsubsection{Inverse dynamic model}

The inverse dynamic model (IDM) of a robot expresses $\tau_{\text {idm }}$ as a function of $q, \dot{q}$ and $\ddot{q}$ (Khalil \& Dombre, 2002). In the case of a linear friction model, the IDM of the EMPS is given by

$\tau_{i d m}=M \ddot{q}+F_{v} \dot{q}+F_{c} \operatorname{sign}(\dot{q})+$ offset.

The important difference between this version of the model and the DDM in (1) is that equation (3) is linear in relation to the dynamic parameters, i.e.,

$\tau_{\text {idm }}=\operatorname{IDM}(q, \dot{q}, \ddot{q}) \boldsymbol{\theta}$,

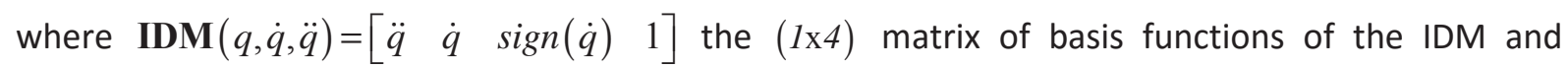
$\boldsymbol{\theta}=\left[\begin{array}{llll}M & F_{v} & F_{c} & \text { offset }\end{array}\right]^{T}$ is the $(4 \times 1)$ vector of the 4 dynamic parameters. This linearity in the unknown parameters makes the IDM relatively easy to estimate using standard statistical methods. This is in contrast to the DDM, which is normally nonlinear with respect to the dynamic parameters and so less straightforward to identify statistically from the experimental data. As a result, it is rarely used for robot identification (Swevers, Verdonck, \& De Schutter, 2007; Gautier, Janot, \& Vandanjon, 2013).

\subsection{Data acquisition and control of the EMPS}

The data available for identification of the EMPS are the measurements $q$ denoted $q_{\text {meas }}$ and the control signal denoted as $v$. The control signal $v$ results from the control law and is linked to $\tau_{\text {idm }}$ by the following relationship 
$\tau_{\text {idm }}=g_{\tau} v ，$

where $g_{\tau}$ is the "drive gain" of the EMPS. Although $g_{\tau}$ is normally provided by the manufacturers, it can be identified using special tests (Gautier \& Briot, 2014). In the case of the EMPS, this yields $g_{\tau}=35.15 \mathrm{~N} / \mathrm{V}$.

As the EMPS is a system involving a pure integrator, it cannot be identified in open loop and so it is first position-controlled by a Proportional-Derivative (PD) controller. In (Gautier, Janot, \& Vandanjon, 2013) it has been shown that a PD control is sufficient to identify the dynamic parameters of robots because excellent tracking is not needed for this purpose. The PD control signal $v$ is given by

$v=K_{p} K_{v}\left(q_{r}-q\right)-K_{v} \dot{q}$,

where $K_{p}$ is the proportional gain and $K_{v}$ is the derivative gain. The calculation of the control gains $K_{p}$ and $K_{v}$ is based on the closed-loop block-diagram for the EMPS, as shown in Fig.2, where $p$ denotes the differentiation operator, while $w_{q}$ denotes the noise on the position.

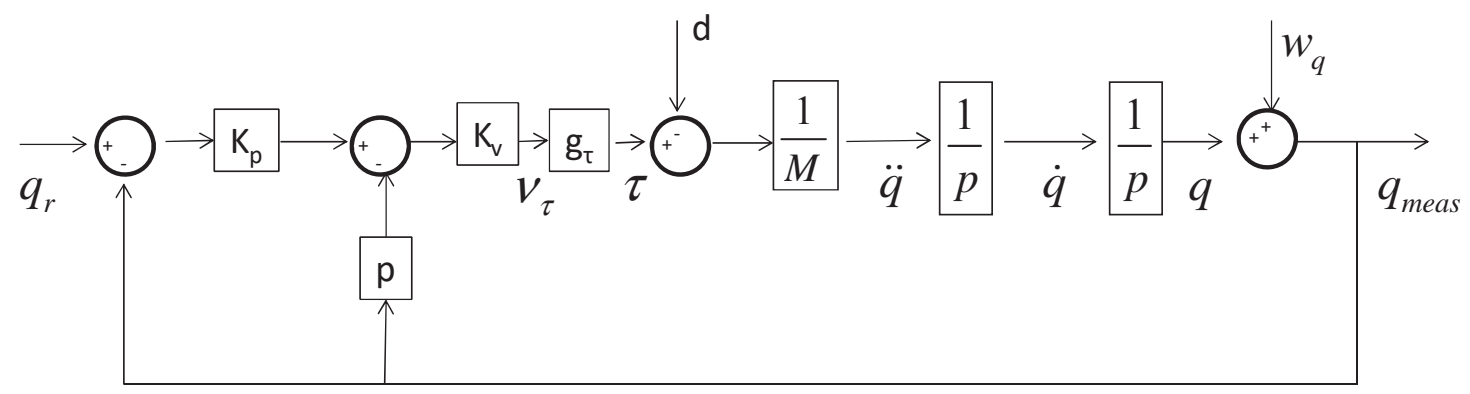

Fig.2. Closed-loop block-diagram for the EMPS prototype

It is assumed that $w_{q}$ is serially independent and homoscedastic, with a bounded variance. These assumptions are usually valid in practice. The EMPS can be modelled as

$q=(\tau-d) / M p^{2}$,

where $d=F_{v} \dot{q}+F_{c} \operatorname{sign}(\dot{q})+$ offset is the linear friction model plus the offset effect, considered as a state-dependent input disturbance. Expression (7) is typical in robotics (and in mechanical engineering in general, see e.g. (Noel, Schoukens, \& Kerschen, 2015)). This explains why such systems are considered as double-integrator systems with a state-dependent perturbation. Naturally, such systems cannot be identified in open loop because they are unstable.

The closed-loop relations are given by

$q=H_{q}(p) q_{r}-H_{d}(p) d$,

with $H_{q}(p)=\frac{g_{\tau} K_{v} K_{p}}{M p^{2}+g_{\tau} K_{v} p+g_{\tau} K_{v} K_{p}}$ and $H_{d}(p)=\frac{1}{M p^{2}+g_{\tau} K_{v} p+g_{\tau} K_{v} K_{p}}$. 
The gains $K_{p}$ and $K_{v}$ are calculated by comparing $H_{q}(p)$ with the following second-order transfer function $H_{q}(p)=1 /\left(\frac{p}{\omega_{n}}\right)^{2}+\frac{2 \eta}{\omega_{n}} p+1$, where $\omega_{n}$ is the natural frequency of the closed loop and $\eta$ is the damping coefficient. This yields $K_{p}=\frac{M \omega_{n}^{2}}{2 \eta \omega_{n} M}$ and $K_{v}=\frac{2 \eta \omega_{n} M}{g_{\tau}}$. With $\omega_{n}=2 \pi \cdot 20 \mathrm{rad} / \mathrm{s}, \eta=1$ selected to avoid overshoot and $M=95 \mathrm{~kg}$ from Computer-Aided-Design (CAD) values, this produces the gain settings $K_{p}=62.831 / \mathrm{s}$ and $K_{v}=679.26 \mathrm{~V} / \mathrm{ms}^{-1}$.

Note that the above simple control design procedure includes approximations, so the design specifications are not met completely in practice. However, this is not important when the resulting experimental data are being used only for identification purposes. More sophisticated nonlinear control system design methods can be exploited after an adequate nonlinear model of the system has been identified. This is discussed later in Section 4.

\subsection{Standard LS-based identification of the EMPS}

As pointed out previously, the traditional identification method developed for robotic systems has been based on the use of the IDM combined with simple linear Least Squares (LS) estimation. However, in this example, we are considering a closed-loop situation and this requires a special approach to identification, see e.g. (Van den Hof, 1998).

First, a pragmatic approach, based on an efficient "tailor-made" data filtering, can be used (see e.g. (Gautier, Janot, \& Vandanjon, 2013)). In (3), $q$ is estimated with its estimate $\hat{q}$ obtained by filtering $q_{\text {meas }}$ through a zero-phase lowpass filter; while $(\hat{\dot{q}}, \hat{\ddot{q}})$ are calculated from $q$ using either a central differentiation algorithm (see e.g. (Gautier, Janot, \& Vandanjon, 2013)); or preferably, as in the present paper, by an optimal filtering algorithm based on recursive fixed interval smoothing (Young et al, 1993) ${ }^{1}$. Hence, the actual motor force $\tau$ differs from $\tau_{i d m}$ by an error $e_{i d m}$ because of model mismatch, noisy measurements and data filtering. The resulting estimation model is then

$$
\tau=\operatorname{IDM}(\hat{q}, \hat{\dot{q}}, \hat{\ddot{q}}) \boldsymbol{\theta}+e .
$$

so that, from $N_{S}$ available samples of the measured signals observed at discrete-time instants while tracking the trajectories $\left(q_{r}, \dot{q}_{r}, \ddot{q}_{r}\right)$, the following over-determined system of regression equations is obtained:

$$
\mathbf{y}_{i d m}=\mathbf{X}_{i d m} \boldsymbol{\theta}+\boldsymbol{\varepsilon}_{i d m},
$$

\footnotetext{
${ }^{1}$ This is available as the IRWSM routine in the CAPTAIN Toolbox for Matlab (see http://captaintoolbox.co.uk/Captain_Toolbox.html/Peter_Young.html).
} 
where $\mathbf{y}_{i d m}$ is the $\left(N_{S} \times 1\right)$ sampled vector of $\tau ; \mathbf{X}_{\text {idm }}$ is the $\left(N_{S} \times 4\right)$ matrix of $\operatorname{IDM}(\hat{q}, \hat{\dot{q}}, \hat{\ddot{q}}) ; \boldsymbol{\varepsilon}_{i d m}$ is the $\left(N_{S} \times 1\right)$ vector of $e_{i d m}$ error terms and $N_{S}$ is the number of samples where the sampling is regular, with a constant sampling interval Ts.

The motor force $\tau$ is perturbed by high-frequency disturbances and, since there is no information on high frequencies variations because the data $(\hat{q}, \hat{\dot{q}}, \hat{q})$ are lowpass filtered, a parallel decimation procedure is used to eliminate torque ripples and any samples at high frequencies that contain no information. By applying the tailor-made data prefiltering, the filtered regression model is assumed to be free of any significant circulatory noise that could lead to biased estimates, so that simple LS can be used to deliver the following estimates

$\hat{\boldsymbol{\theta}}_{L S}=\left(\mathbf{X}_{i d m}^{T} \mathbf{X}_{i d m}\right)^{-1} \mathbf{X}_{i d m}^{T} \mathbf{y}_{i d m}$.

The identifiability of the LS solution (11) is ensured if $\mathbf{X}_{\text {idm }}$ is a column-full-rank matrix i.e. $\operatorname{rank}\left(\mathbf{X}_{i d m}\right)=4$ and this requires that the trajectories $\left(q_{r}, \dot{q}_{r}, \ddot{q}_{r}\right)$ are sufficiently exciting.

Provided the LS identification residuals are zero mean and white (serially uncorrelated), and it is assumed that $\mathbf{X}_{i d m}$ is deterministic, then the covariance matrix of the LS estimates can be calculated as follows using standard linear regression theory (see e.g. Young, 2011 and Janot, Vandanjon, \& Gautier, 2014a):

$\boldsymbol{\Sigma}_{L S}=\hat{\sigma}_{\varepsilon_{i d m}^{2}}\left(\mathbf{X}_{i d m}^{T} \mathbf{X}_{i d m}\right)^{-1}$,

where $\hat{\sigma}_{\varepsilon_{i d m}}^{2}=\left\|\mathbf{y}_{i d m}-\mathbf{X}_{i d m} \hat{\boldsymbol{\theta}}_{L S}\right\| /\left(N_{S}-4\right)$.

$\hat{\sigma}_{\hat{\theta}_{L S(i)}}^{2}=\Sigma_{L S}(i, i)$ is the $i^{\text {th }}$ diagonal coefficient of $\Sigma_{L S}$. The relative standard deviation $\% \sigma_{\hat{\theta}_{L S(i)}}$ is given by $\% \hat{\sigma}_{\hat{\theta}_{L S(i)}}=100 \hat{\sigma}_{\hat{\theta}_{L S(i)}} /\left|\hat{\theta}_{L S}(i)\right|$ for $\left|\hat{\theta}_{L S}(i)\right| \neq 0$.

Note that the statistical assumptions required for these results to apply are met in the present practical context thanks to the accurate experimental data and appropriate data filtering (see Janot, Vandanjon, \& Gautier, 2014a) and (Brunot et al., 2015). However, if this filtering is not adequate and the noise level is too high, then the LS estimation would need to be replaced, for instance, by the instrumental variable approach presented in (Janot, Vandanjon, \& Gautier, 2014a).

\subsection{SDP-based identification method of the EMPS}

As stated in section 2.2, the linear friction model (2) is only valid within a given velocity range. At low velocities, the friction normally exhibits clear non-linear effects (e.g. Stiction and Stribeck etc.). It is convenient, therefore, to introduce a state-dependent parameter that is able to cope with such nonlinearities. Also, in order to validate/invalidate the assumption that the other dynamic parameters are time-invariant, other state-dependent parameters may be identified during SDP estimation. 
In the case of the EMPS, the mass $M$ may be acceleration-dependent. The IDM is thus rewritten as

$$
\tau_{\text {idm }}=M(\ddot{q}) \ddot{q}+d_{\text {fric }}(\dot{q}),
$$

with $d_{\text {fric }}(\dot{q})=\tau_{\text {fric }}$ and $M(\ddot{q})$ allowing for the possibility of any significant acceleration dependency. Note that $d_{\text {fric }}(\dot{q})$ is simply the friction force that depends only on the velocity and so it can be considered, therefore, as a state-dependent parameter $\left(d_{\text {fric }}(\dot{q})\right.$ is used instead of $d(\dot{q})$ in order to avoid ambiguity with the linear friction model).

The IDM (13) is now written as a linear-in-the-state-dependent-parameters form given by

$$
\tau_{i d m}=\mathbf{I D M}_{s d p}(q, \dot{q}, \ddot{q}) \boldsymbol{\theta}_{s d p},
$$

with $\mathbf{I D M}_{s d p}(q, \dot{q}, \ddot{q})=\left[\begin{array}{ll}\ddot{q} & 1\end{array}\right]$ and $\boldsymbol{\theta}_{s d p}=\left[M(\ddot{q}) \quad d_{\text {fric }}(\dot{q})\right]^{T}$.

As with the IDIM-LS method, the actual force $\tau$ differs from $\tau_{\text {idm }}$ by an error $e_{\text {sdp }}$ and so, in a similar fashion, the following over-determined system of equations is obtained

$\mathbf{y}_{i d m}=\mathbf{X}_{s d p}(\hat{q}, \hat{\dot{q}}, \hat{q}) \boldsymbol{\theta}_{s d p}+\boldsymbol{\varepsilon}_{s d p}$,

where $\mathbf{X}_{s d p}$ is the $\left(N_{S} \times 2\right)$ sampled matrix of $\operatorname{IDM}_{s d p}(\hat{q}, \hat{\dot{q}}, \hat{\ddot{q}}) ; \boldsymbol{\varepsilon}_{s d p}$ is the $\left(N_{S} \times 1\right)$ sampled vector of $e_{s d p}$ and $\hat{q}, \hat{\dot{q}}, \hat{\dot{q}}$ are constructed as explained in Section 2.4.

The acceleration-dependent mass $M(\ddot{q}(t))$ and the friction nonlinearity $d_{\text {fric }}(\dot{q}(t))$ are simultaneously estimated by the SDP routine in the CAPTAIN Toolbox. The SDP routine provides $\hat{\mathbf{M}}(\hat{\ddot{q}})$, the estimate of $\mathbf{M}(\hat{\ddot{q}})$, the $\left(N_{S} \times 1\right)$ sampled vector of the acceleration-dependent mass $M(\hat{\ddot{q}})$; and $\hat{\mathbf{d}}_{\text {fric }}(\hat{\dot{q}})$, the estimate of $\mathbf{d}_{\text {fric }}(\hat{\dot{q}})$, the $\left(N_{S} \times 1\right)$ sampled vector of the velocity-dependent friction $d_{\text {fric }}$. As a result, the SDP model residual, $\hat{\boldsymbol{\varepsilon}}_{\text {sdp }}$, is calculated as

$\hat{\boldsymbol{\varepsilon}}_{s d p}=\mathbf{y}_{i d m}-{ }^{d i a g} \mathbf{X}_{s d p} \hat{\boldsymbol{\Theta}}_{s d p}$,

where ${ }^{\operatorname{diag}} \mathbf{X}_{\text {sdp }}=\left[\operatorname{diag}(\hat{\ddot{\mathbf{q}}}) \quad \mathbf{I}_{N_{S}}\right]$ is the $\left(N_{S} \times 2 \cdot N_{S}\right)$ matrix of $\mathbf{X}_{s d p}(\hat{q}, \hat{\dot{q}}, \hat{\ddot{q}})$ all of whose sampled basis functions are diagonalized and horizontally stacked; $\operatorname{diag}(\hat{\hat{\mathbf{q}}})$ is the $\left(N_{S} \times N_{S}\right)$ diagonal matrix whose the $\mathrm{i}^{\text {th }}$ element is the $\mathrm{i}^{\text {th }}$ element of $\hat{\ddot{\mathbf{q}}}$ the $\left(N_{S} \times 1\right)$ sampled vector of $\hat{\ddot{q}} ; \mathbf{I}_{N_{S}}$ is the $\left(N_{S} \times N_{S}\right)$ identity matrix; and $\hat{\boldsymbol{\Theta}}_{\text {sdp }}=\left[\begin{array}{ll}\hat{\mathbf{M}}(\hat{\ddot{q}})^{T} & \hat{\mathbf{d}}_{\text {fric }}(\hat{\dot{q}})^{T}\end{array}\right]^{T}$ is the estimate of $\boldsymbol{\Theta}_{\text {sdp }}=\left[\begin{array}{ll}\mathbf{M}(\hat{q})^{T} & \mathbf{d}_{\text {fric }}(\hat{\dot{q}})^{T}\end{array}\right]^{T}$ the $\left(2 \cdot N_{S} \times 1\right)$ sampled vector of $\boldsymbol{\theta}_{\text {sdp }}$. Finally, the relative error is given by $\left\|\hat{\boldsymbol{\varepsilon}}_{s d p}\right\| /\left\|\mathbf{y}_{\text {idm }}\right\|$. 


\subsection{Experimental results}

The dynamic parameters $M, F_{v}, F_{c}$ and offset are first identified with the standard identification IDIM-LS approach described in section 2.4 .

As pointed out in section 2.4, since it is possible to generate very accurate experimental data and utilize appropriate data filtering, the LS estimates can be considered as unbiased, even though the EMPS is identified in closed loop. This point is dealt with in (Janot, Vandanjon, \& Gautier, 2014a) and (Brunot et al., 2015). The LS estimates and the relative errors are given in Table 1.

The acceleration-dependent mass estimated by the SDP method is illustrated in Fig.3. We see that the SDP estimation suggests a constant value very similar to the IDIM-LS estimate (there is only a difference of $60 \mathrm{~g}$ which is negligible compared with $95 \mathrm{Kg}$ ). Note also that the optimized Noise Variance Ratio (NVR) associated with the $\hat{\mathbf{M}}(\hat{\ddot{q}})$ term in the SDP regression, which defines the amount of state dependency (see Young 2011), is 1.0e-23 i.e. virtually zero; while the NVR associated with $\hat{\mathbf{d}}_{\text {fric }}(\hat{\dot{q}})$ is 2.9. This large difference between the two NVR's is consistent with our a priori knowledge and suggests that the mass is not acceleration-dependent. As similar results are obtained with a position- and velocity-dependent mass i.e. $M(q)$ and $M(\dot{q})$, respectively, it can be assumed that the mass is state-invariant. Given the large value of 2.9 for the NVR associated with the friction SDP estimate, the SDP method is able to reconstruct the shape of the frictional nonlinearity, as shown in Fig.4. Finally, the relative error obtained with the SDP-based identification method is only $1.5 \%$.

At first glance, the results obtained with the standard IDIM-LS identification method and the linear friction model seems quite acceptable. Indeed, the relative error is small (less than $5 \%$ ) and the estimated mass is close to its CAD value i.e. $95 \mathrm{~kg}$. However, the relative error obtained using SDP estimation is only $1.5 \%$ and we need to examine the reason for this discrepancy between the results. This is due to the estimates of the friction parameters, as revealed in Fig.4. Here we see that there is a small but sustained difference between the red and blue lines in the lower part of the curves (negative velocities), which suggests that there could be a small bias in the latter (see the enlarged panel in the lower right corner of Fig.4). In other words, there is a small error in the friction model identified by the standard method and the SDP friction estimate eliminates this by suggesting an asymmetrical friction model; i.e. a model that depends on the sign of $\dot{q}$ where, for negative velocities, the red and blue lines are not perfectly parallel. This asymmetry can be explained by the fatigue of the screw.

In order to take this asymmetry into account, the friction model is modified to

$$
\tau_{\text {fric }}=F_{v}^{+} 0^{+}(\dot{q})+F_{c}^{+} \operatorname{sign}\left(0^{+}(\dot{q})\right)+F_{v}^{-} 0^{-}(\dot{q})+F_{c}^{-} \operatorname{sign}\left(0^{-}(\dot{q})\right) \text {, }
$$

where $0^{+}$and $0^{-}$are two operators defined by $0^{+}(\dot{q})=\dot{q}\left(\frac{1+\operatorname{sign}(\dot{q})}{2}\right)$ and $0^{-}(\dot{q})=\dot{q}\left(\frac{1-\operatorname{sign}(\dot{q})}{2}\right)$; $F_{v}^{+}$and $F_{c}^{+}$(resp. $F_{v}^{-}$and $F_{c}^{-}$) are the viscous and Coulomb friction coefficients for the positive (resp. negative) velocities. Finally, $0^{+}(\dot{q})$ (resp. $0^{-}(\dot{q})$ ) returns $\dot{q}$ if $\dot{q}>0$ (resp. $\dot{q}<0$ ) and 0 otherwise. 
When equation (17) is inserted into (1), it yields the following linear-in-the-parameters IDM

$\tau_{\text {idm }}=\mathbf{I D M}_{\text {asym }} \boldsymbol{\theta}_{\text {asym }}$,

with $\mathbf{I D M}_{\text {asym }}=\left[\begin{array}{lllll}\ddot{q} & 0^{+}(\dot{q}) & \operatorname{sign}\left(0^{+}(\dot{q})\right) & 0^{-}(\dot{q}) & \operatorname{sign}\left(0^{-}(\dot{q})\right)\end{array}\right]$ and $\boldsymbol{\theta}_{\text {asym }}=\left[\begin{array}{lllll}M & F_{v}^{+} & F_{c}^{+} & F_{v}^{-} & F_{c}^{-}\end{array}\right]^{T}$.

As in the previous situations, the actual force $\tau$ differs from $\tau_{\text {idm }}$ by an error $e_{\text {asym }}$ and the resulting over-determined set of equations takes the form,

$\mathbf{y}_{\text {idm }}=\mathbf{X}_{\text {asym }} \boldsymbol{\theta}_{\text {asym }}+\boldsymbol{\varepsilon}_{\text {asym }}$,

where $\mathbf{y}_{\text {idm }}$ is the $\left(N_{S} \times 1\right)$ sampled vector of $\tau ; \mathbf{X}_{\text {asym }}$ is the $\left(N_{S} \times 5\right)$ matrix of $\operatorname{IDM}_{\text {asym }}(\hat{q}, \hat{\dot{q}}, \hat{q})$; and $\boldsymbol{\varepsilon}_{\text {asym }}$ is the $\left(N_{S} \times 1\right)$ vector of $e_{\text {asym }}$ error terms. The LS estimates of (19) and their associated deviations are given by (11) and (12), $\mathbf{X}_{i d m}$ being replaced with $\mathbf{X}_{\text {asym }}$.

The resulting estimates and the relative error are given in Table 2. These confirm that the friction has asymmetric behaviour because $F_{v}^{+}$is significantly different from $F_{v}^{-}$, while the estimate of $M$ has not changed. Furthermore, the LS relative error has now decreased to $1.5 \%$, a value that is compatible with the relative error obtained with the non-parametric SDP method. The direct comparison plotted in Fig. 5 shows clearly that the agreement between the SDP estimated friction shape and the asymmetrical friction model reconstructed with the above LS estimates is now acceptable. This finally estimated relationship is the parameterised SDP model of the EMPS, which we will term the IDIMSDP model. Clearly, if the prior assumptions of the IDIM-LS estimation are modified in the light of the SDP estimation, then the IDIM-LS estimation results would be the same.

Table 1 : IDIM-LS estimates of the EMPS with the standard linear friction model

\begin{tabular}{|c|c|}
\hline Parameters & IDIM-LS estimates $\left(\% \sigma_{\hat{\theta}_{L S(i)}}\right)$ \\
\hline$M(\mathrm{~kg})$ & $95.08(0.15 \%)$ \\
\hline$F_{v}\left(\mathrm{~N} / \mathrm{ms}^{-1}\right)$ & $202.30(0.74 \%)$ \\
\hline$F_{c}(\mathrm{~N})$ & $20.53(0.64 \%)$ \\
\hline offset $(\mathrm{N})$ & $-3.19(1.81 \%)$ \\
\hline Relative error & $3.7 \%$ \\
\hline
\end{tabular}


Table 2 : Parametric IDIM-SDP estimates for an asymmetrical friction model

\begin{tabular}{|c|c|}
\hline Parameters & LS estimates $\left(\% \sigma_{\hat{\theta}_{L S(i)}}\right)$ \\
\hline$M(\mathrm{~kg})$ & $95.12(0.11 \%)$ \\
\hline$F_{v}^{+}\left(\mathrm{N} / \mathrm{ms}^{-1}\right)$ & $165.80(0.92 \%)$ \\
\hline$F_{c}^{+}(\mathrm{N})$ & $20.19(0.67 \%)$ \\
\hline$F_{v}^{-}\left(\mathrm{N} / \mathrm{ms}^{-1}\right)$ & $238.89(0.64 \%)$ \\
\hline$F_{c}^{-}(\mathrm{N})$ & $20.85(0.65 \%)$ \\
\hline Relative error & $1.5 \%$ \\
\hline
\end{tabular}

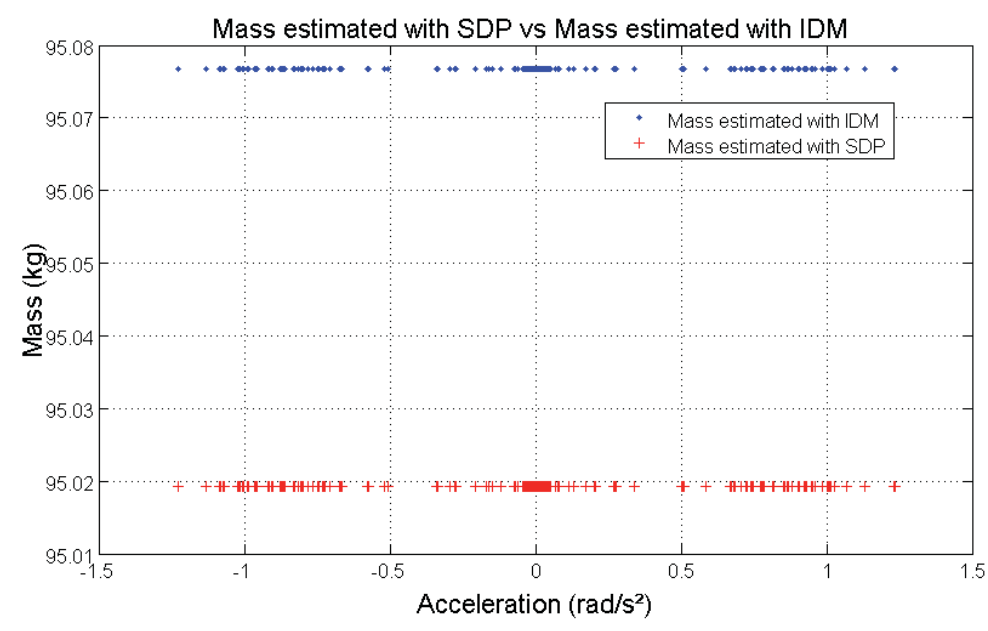

Fig.3: Direct comparison between mass estimated with the IDIM-LS method (blue dots) and the acceleration-dependent mass estimated with the SDP algorithm (red crosses): it is clear that the mass is acceleration-independent. 

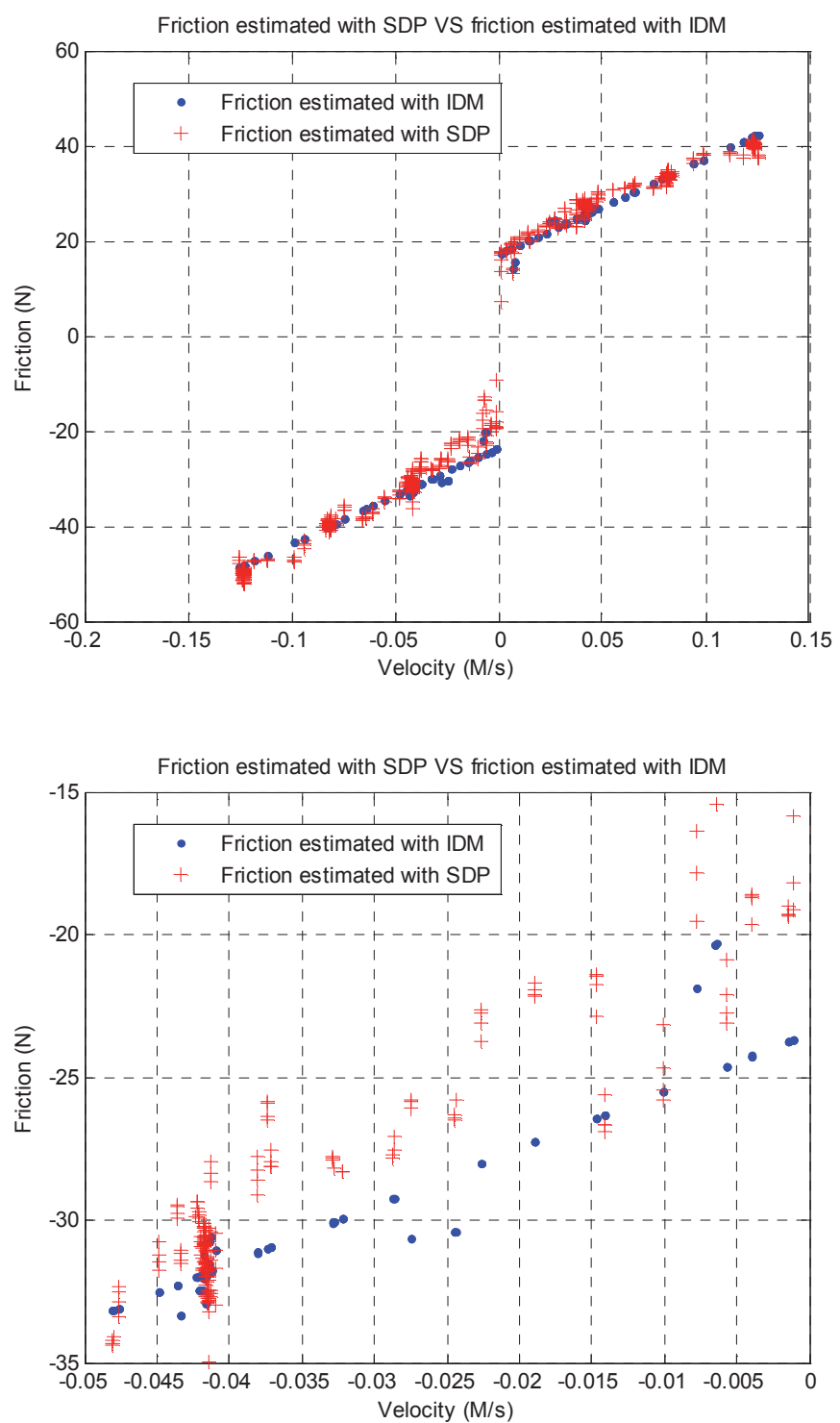

Fig.4. The upper panel shows a direct comparison between the friction nonlinearity reconstructed with the LS estimates of the linear friction model (blue dots) and the nonlinearity estimated by the SDP algorithm (red crosses). The enlarged portion shown in the lower panel reveals a small but persistent error that suggests an asymmetrical friction model. 


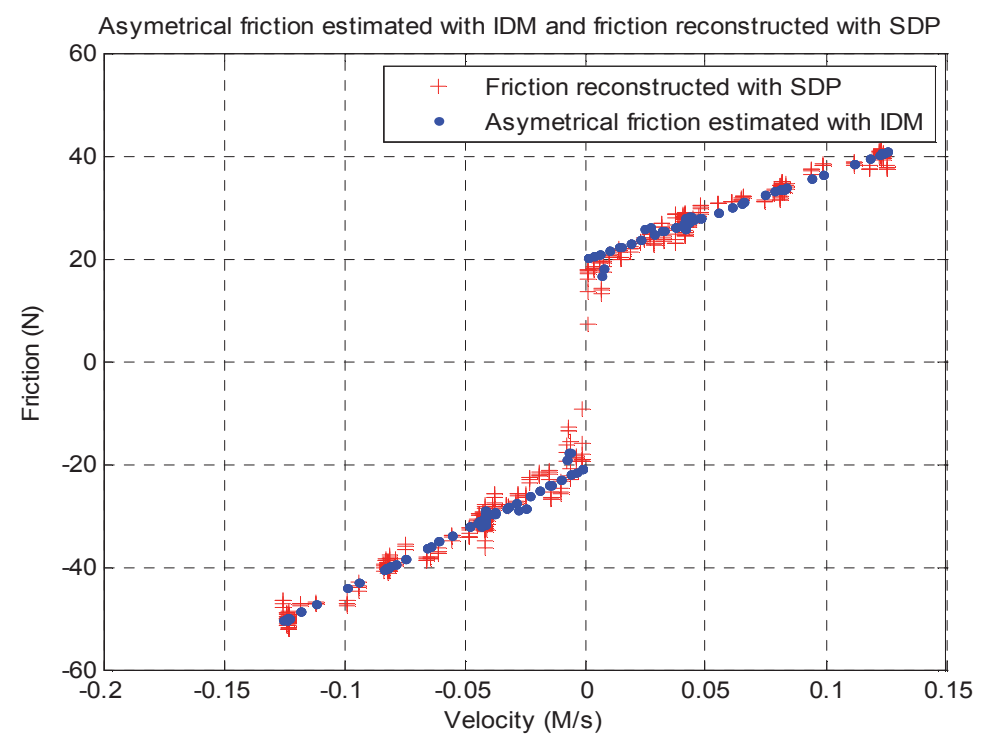

Fig.5. Direct comparison between the friction nonlinearity estimated with the asymmetrical linear friction model (2nd stage IDIM-SDP model, blue dots) and the friction nonlinearity previously estimated by the first stage SDP algorithm (red crosses), showing that the two estimates are consistent and confirm the asymmetry.

\section{Second case study: TX40 robot}

\subsection{Introduction and presentation of the TX40 robot}

In the previous section, it has been shown that the SDP method can be used as a two-stage alternative to the IDIM-LS method for estimating and evaluating the quality of the friction model; an alternative that helps to avoid over-reliance on prior conceptions about the nature of the nonlinear characteristics. In this section, SDP estimation is evaluated on a more challenging system: the TX40 robot.

The Stäubli TX40 robot has a serial structure with six rotational joints. Its kinematics are defined by the DHM notation, as in Fig.6 (Khalil \& Dombre, 2002). The geometric parameters defining the TX40 frames are given in Table 3: $\sigma_{j}=0$ means that joint $j$ is rotational; $\alpha_{j}$ and $d_{j}$ give, respectively, the angle and the distance between $z_{j-1}$ and $z_{j}$ along $x_{j-1} ; \theta_{j}$ and $r_{j}$ give, respectively, the angle and the distance between $x_{j-1}$ and $x_{j}$ along $z_{j}$.

The joint positions and the control signals are stored with a measurement frequency $f_{m}=5 \mathrm{kHz}$. The Reference trajectories are fifth order polynomials that excite the base parameters sufficiently for identification purposes. 
Table 3: Geometric parameters of the TX40 robot

\begin{tabular}{|c|c|c|c|c|c|}
\hline $\mathrm{j}$ & $\sigma_{\mathrm{j}}$ & $\alpha_{\mathrm{j}}$ & $\mathrm{d}_{\mathrm{j}}$ & $\theta_{\mathrm{j}}$ & $\mathrm{r}_{\mathrm{j}}$ \\
\hline 1 & 0 & 0 & 0 & $\theta_{1}$ & 0 \\
\hline 2 & 0 & $-\pi / 2$ & 0 & $\theta_{2}$ & 0 \\
\hline 3 & 0 & 0 & $\mathrm{~d}_{3}=0.225 \mathrm{~m}$ & $\theta_{3}$ & $\mathrm{r}_{3}=0.035 \mathrm{~m}$ \\
\hline 4 & 0 & $\pi / 2$ & 0 & $\theta_{4}$ & $\mathrm{r}_{4}=0.225 \mathrm{~m}$ \\
\hline 5 & 0 & $-\pi / 2$ & 0 & $\theta_{5}$ & 0 \\
\hline 6 & 0 & $\pi / 2$ & 0 & $\theta_{6}$ & 0 \\
\hline
\end{tabular}
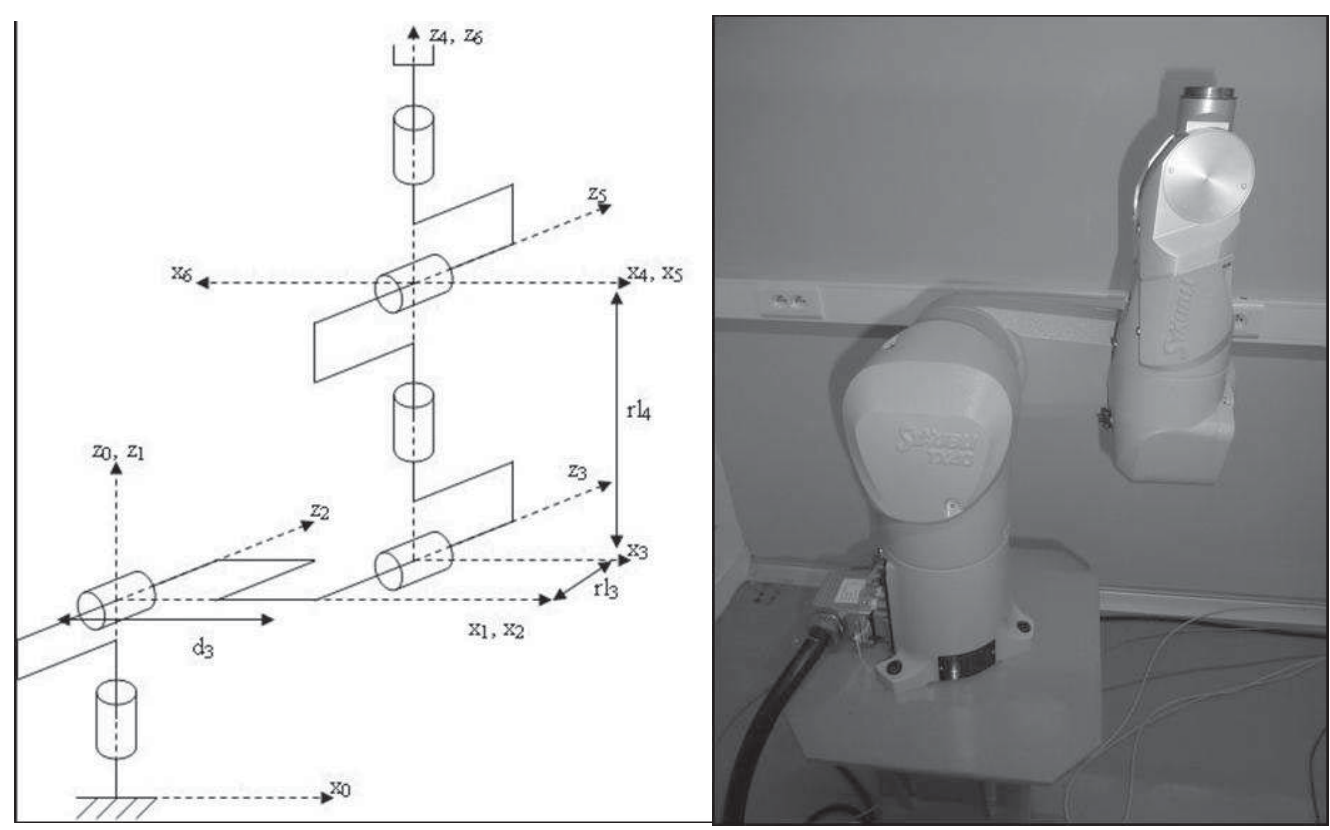

Fig.6: Link frames of TX40 Stäubli robot

\subsection{Inverse dynamic model of the second link with the usual method}

When only the second link is moving, with the others maintained at their steady-sate levels, the IDM of the second link (also known as the arm of the robot) reduces to

$$
\tau_{\text {idm }_{2}}=\mathrm{ZZ}_{2} \ddot{q}_{2}+\tau_{\text {grav }_{2}}+\tau_{\text {fric }_{2}}+\text { offset }_{2},
$$

where $\tau_{i d m_{2}}$ is the second joint torque; $\tau_{\text {grav }_{2}}$ is the gravity torque of the second link given by $\tau_{\text {grav }_{2}}=-g M X_{2} \cos \left(q_{2}\right)+g M Y_{2} \sin \left(q_{2}\right), \quad M X_{2}$ and $M Y_{2}$ being the components of the gravity effect; $g=9.81 \mathrm{~m} / \mathrm{s}^{2}$ is the gravity constant; $\tau_{\text {fric }}$ is the friction torque of the second link; $q_{2}, \dot{q}_{2}$ and $\ddot{q}_{2}$, are, respectively, the position, velocity and acceleration of the second link; $Z Z Z_{2}$ is the total inertia of the second link; and offset ${ }_{2}$ is an offset parameter.

In the case of a linear friction model, $\tau_{\text {fric }}$ is given by 
$\tau_{\text {fric }}=F_{v 2} \dot{q}_{2}+F_{c 2} \operatorname{sign}\left(\dot{q}_{2}\right)$,

where $F_{v 2}$ and $F_{c 2}$ are the viscous and Coulomb friction parameters of the second link.

The resulting IDM is linear in relation to the dynamic parameters, i.e.,

$\tau_{i d m_{2}}=\mathbf{I D M}\left(q_{2}, \dot{q}_{2}, \ddot{q}_{2}\right) \boldsymbol{\theta}$,

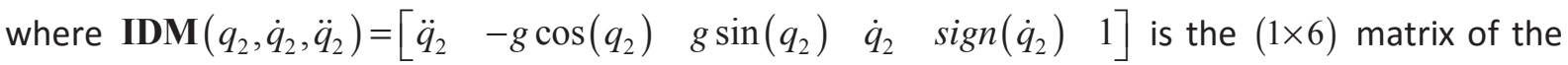
basis functions of the IDM and $\boldsymbol{\theta}=\left[\begin{array}{llllll}Z_{2} & M X_{2} & M Y_{2} & F_{v 2} & F_{c 2} & \text { offset }_{2}\end{array}\right]^{T}$ is the $(6 \times 1)$ vector of the dynamic parameters. As $\tau_{2}$ differs from $\tau_{\text {idm }}$ by an error $e_{i d m_{2}}$ and there are $N_{S}$ available samples of the measured signals, it is straightforward to formulate the following over-determined system of equations

$\mathbf{y}_{\text {idm }}=\mathbf{X}_{i d m_{2}} \boldsymbol{\theta}+\boldsymbol{\varepsilon}_{i d m_{2}}$,

where $\mathbf{y}_{i d m_{2}}$ is the $\left(N_{S} \times 1\right)$ sampled vector of $\tau_{2} ; \mathbf{X}_{i d m_{2}}$ is the $\left(N_{S} \times 6\right)$ matrix of $\operatorname{IDM}\left(\hat{q}_{2}, \hat{\dot{q}}_{2}, \hat{\ddot{q}}_{2}\right) ; \boldsymbol{\varepsilon}_{i d m_{2}}$ is the $\left(N_{S} \times 1\right)$ vector of $e_{i d m_{2}}$ error terms; and $\hat{q}_{2}, \hat{\dot{q}}_{2}, \hat{\vec{q}}_{2}$ are constructed as explained in section 2.4. The LS estimates from equation (19) and their associated covariance matrix are given by (11) and (12) , with $\mathbf{X}_{i d m}$ being replaced with $\mathbf{X}_{i d m_{2}}$, again under the assumption that the prefiltering has been fully effective in its removal of noise from the variables.

\subsection{Using the SDP function of the CAPTAIN Toolbox to retrieve the shapes of gravity and friction}

When using the SDP estimation method, the IDM is rewritten in the form

$\tau_{i d m_{2}}=Z Z_{2} \ddot{q}_{2}+d\left(q_{2}, \dot{q}_{2}\right)$,

with $\quad d\left(q_{2}, \dot{q}_{2}\right)=d_{q_{2}}\left(q_{2}\right)+d_{\dot{q}_{2}}\left(\dot{q}_{2}\right) \quad$ where $\quad d_{q_{2}}\left(q_{2}\right)=-g M X_{2} \cos \left(q_{2}\right)+g M Y_{2} \sin \left(q_{2}\right) \quad$ and $d_{\dot{q}_{2}}\left(\dot{q}_{2}\right)=F_{v 2} \dot{q}_{2}+F_{c 2} \operatorname{sign}\left(\dot{q}_{2}\right)+$ offset $_{2}$.

It is assumed here that the parameter $d\left(q_{2}, \dot{q}_{2}\right)$ depends on the position $q_{2}$ and the velocity $\dot{q}_{2}$ so, ideally, it should be identified using the Multi-SDP method, (see e.g. Sadeghi et al., 2010). Unfortunately, such a multi-state dependent algorithm is quite involved and has not yet been fully implemented in the CAPTAIN Toolbox. As a result, the existing SDP routine in CAPTAIN cannot be used directly in this situation. This difficulty has been partially circumvented, however, by developing an additional iterative "back-fitting" procedure, which is quite similar to that used in the standard SDP algorithm. Provided it converges satisfactorily, back-fitting estimation such as this is reasonably justified in this example because the perturbations can be considered as decoupled: i.e. one depends on the position alone, while the other depends on the velocity alone, so the estimation is potentially "separable". 
As in the case of IDIM-LS estimation, $\tau_{2}$ differs from $\tau_{\text {idm }}$ by an error $e_{s d p_{2}}$ and so, from $N_{S}$ available samples, the following system of regression equations is obtained

$\mathbf{y}_{\text {idm }}=Z Z Z_{2} \hat{\overrightarrow{\mathbf{q}}}_{2}+\mathbf{d}\left(\hat{q}_{2}, \hat{\dot{q}}_{2}\right)+\boldsymbol{\varepsilon}_{s d p_{2}}$,

where $\mathbf{d}\left(\hat{q}_{2}, \hat{\dot{q}}_{2}\right)$ is the $\left(N_{S} \times 1\right)$ sampled vector of $d\left(\hat{q}_{2}, \hat{\dot{q}}_{2}\right) ; \boldsymbol{\varepsilon}_{s d p_{2}}$ is the $\left(N_{S} \times 1\right)$ sampled vector of $e_{s d p_{2}} ; \hat{q}_{2}, \hat{\dot{q}}_{2}, \hat{\ddot{q}}_{2}$ are constructed as explained in Section 2.4.; and $\hat{\ddot{q}}_{2}$ is the $\left(N_{S} \times 1\right)$ sampled vector of $\hat{\ddot{q}}_{2}$. The SDP iterations then involve the following, three step procedure, with steps 2 to 3 repeated until convergence is achieved:

1. Initial Step: the estimate of $\mathbf{d}\left(\hat{q}_{2}, \hat{\dot{q}}_{2}\right)$, denoted by $\hat{\mathbf{d}}\left(\hat{q}_{2}, \hat{\dot{q}}_{2}\right)$, is calculated as follows

$\hat{\mathbf{d}}\left(\hat{q}_{2}, \hat{\dot{q}}_{2}\right)=\mathbf{y}_{\text {idm }_{2}}-\widehat{Z Z}_{2} \ddot{\mathbf{q}}_{2}$,

where $\widehat{Z Z} \widehat{Z Z}_{2}$ is the CAD value of $Z Z_{2}$. Then, the shapes of $\mathbf{d}_{q_{2}}\left(q_{2}\right)$ and $\mathbf{d}_{\dot{q}_{2}}\left(\dot{q}_{2}\right)$ are initialized with $\hat{\mathbf{d}}_{q_{2}}^{0}\left(\hat{q}_{2}\right)$ and $\hat{\mathbf{d}}_{\dot{q}_{2}}^{0}\left(\hat{\dot{q}}_{2}\right)=\mathbf{0}$, respectively, since they are assumed to be unknown to the users.

for $k=1,2, \ldots$, until convergence

At each step $k$,

2. the estimate of $\mathbf{d}_{q_{2}}^{k}\left(\hat{q}_{2}\right)$, denoted by $\hat{\mathbf{d}}_{q_{2}}^{k}\left(\hat{q}_{2}\right)$, is estimated using the SDP algorithm: here, the measurement vector is $\mathbf{y}_{q_{2}}^{k}=\hat{\mathbf{d}}\left(\hat{q}_{2}, \hat{\dot{q}}_{2}\right)-\hat{\mathbf{d}}_{\dot{q}_{2}}^{k}\left(\hat{\dot{q}}_{2}\right)$; the regressor is $\mathbf{z}=\mathbf{1}$; and the state vector is $\mathbf{x}=\hat{\mathbf{q}}_{2}, \hat{\mathbf{q}}_{2}$, being the $\left(N_{S} \times 1\right)$ sampled vector of $\hat{q}_{2}$.

3. the estimate of $\mathbf{d}_{\dot{q}_{2}}^{k}\left(\hat{\dot{q}}_{2}\right)$, denoted by $\hat{\mathbf{d}}_{\dot{q}_{2}}^{k}\left(\hat{\dot{q}}_{2}\right)$, is estimated using the SDP algorithm: here, the measurement vector is $\mathbf{y}_{\dot{q}_{2}}^{k}=\hat{\mathbf{d}}\left(\hat{q}_{2}, \hat{\dot{q}}_{2}\right)-\hat{\mathbf{d}}_{q_{2}}^{k}\left(\hat{q}_{2}\right)$; the regressor is again $\mathbf{z}=\mathbf{1}$; but the state vector is now $\mathbf{x}=\hat{\dot{\mathbf{q}}}_{2}, \hat{\dot{\mathbf{q}}}_{2}$, being the $\left(N_{S} \times 1\right)$ sampled vector of $\hat{\dot{q}}_{2}$.

end

The following convergence criterion has been found to yield good results

$\frac{\left\|\hat{\mathbf{d}}^{k}\left(\hat{q}_{2}, \hat{\dot{q}}_{2}\right)\right\|-\left\|\hat{\mathbf{d}}^{k-1}\left(\hat{q}_{2}, \hat{\dot{q}}_{2}\right)\right\|}{\left\|\hat{\mathbf{d}}^{k}\left(\hat{q}_{2}, \hat{\dot{q}}_{2}\right)\right\|} \leq$ tol,

where $\hat{\mathbf{d}}^{k}\left(\hat{q}_{2}, \hat{\dot{q}}_{2}\right)=\hat{\mathbf{d}}_{q_{2}}^{k}\left(\hat{q}_{2}\right)+\hat{\mathbf{d}}_{\dot{q}_{2}}^{k}\left(\hat{\dot{q}}_{2}\right)$ is the estimate of $\mathbf{d}^{k}\left(\hat{q}_{2}, \hat{\dot{q}}_{2}\right)=\mathbf{d}_{q_{2}}^{k}\left(\hat{q}_{2}\right)+\mathbf{d}_{\dot{q}_{2}}^{k}\left(\hat{\dot{q}}_{2}\right)$ at step $k$ and tol is a threshold defined by the users (between $0.5 \%$ and $5.0 \%$ ). Finally, the relative error is simply given by $\left\|\hat{\boldsymbol{\varepsilon}}_{\text {sdp }}\right\| /\left\|\mathbf{y}_{\text {idm }}\right\|$ with $\hat{\boldsymbol{\varepsilon}}_{\text {sdp }}=\mathbf{y}_{\text {idm }}-\widehat{Z Z}_{2} \ddot{\mathbf{q}}_{2}-\hat{\mathbf{d}}^{k}\left(\hat{q}_{2}, \hat{\dot{q}}_{2}\right)$.

Although this back-fitting procedure is reasonably justified in this example, caution is still necessary because gravity and friction are low-frequency phenomena, so that it is not clear a priori that the SDP 
algorithm will be able to extract $d_{q_{2}}\left(q_{2}\right)$ and $d_{\dot{q}_{2}}\left(\dot{q}_{2}\right)$ from $d\left(q_{2}, \dot{q}_{2}\right)$ in a completely separable manner. However, as we see below, it does work reasonably well in this example when only the second link is moving.

\subsection{Experimental results}

\subsubsection{Only the second link is moving}

The dynamic parameters are first identified with the IDIM-LS method with only the second link being excited by the fifth-order polynomial trajectories that are required to ensure good estimation of the dynamic characteristics. The other links are maintained at their steady-state levels.

The IDIM-LS estimates are given in Table 4. The reference values are the CAD values for the inertia and gravity parameters; and the friction parameters are the estimated values given in (Janot, Vandanjon, \& Gautier, 2014a). The reconstruction is quite good with a relative error of $5.8 \%$. Finally, the estimates of inertia, gravity and friction parameters are close to the reference values.

The iterative-SDP estimation procedure outlined above in section 3.3 is initialized with $\hat{\mathbf{d}}_{q_{2}}^{0}\left(\hat{q}_{2}\right)$ and $\hat{\mathbf{d}}_{\dot{q}_{2}}^{0}\left(\hat{\dot{q}}_{2}\right)=\mathbf{0}$, while tol $=1 \%$ is used as the convergence criterion. In order to evaluate the resulting estimates, the SDP nonlinearities $\hat{\mathbf{d}}_{q_{2}}^{k}\left(\hat{q}_{2}\right)$ and $\hat{\mathbf{d}}_{\dot{q}_{2}}^{k}\left(\hat{\dot{q}}_{2}\right)$ identified by this procedure are regressed on $\left[\begin{array}{lll}-g \cos \left(\hat{q}_{2}\right) & g \sin \left(\hat{q}_{2}\right) & 1\end{array}\right]$ and $\left[\begin{array}{ll}\hat{\dot{q}}_{2} & \operatorname{sign}\left(\hat{\dot{q}}_{2}\right)\end{array}\right]$, respectively, using standard linear least squares estimation. These constant parameter LS estimates are referred to as the IDIM-SDP estimates in Table 4 and one would expect these to be close to the IDIM-LS estimates if the SDP method is to be considered successful and the SDP identified nonlinearities $\hat{\mathbf{d}}_{q_{2}}^{k}\left(\hat{q}_{2}\right)$ and $\hat{\mathbf{d}}_{\dot{q}_{2}}^{k}\left(\hat{\dot{q}}_{2}\right)$ are to be trusted. In this case, the SDP iterative algorithm converges in 5 iterations and the results plotted in Fig.7, together with the parameter estimates given in Table 4, demonstrate that the iterative SDP algorithm does indeed yield very good results in this example. In particular, the shape of the gravity and friction nonlinearities reconstructed by the SDP-based algorithm match the shape of the same nonlinearities reconstructed with the IDIM-LS estimates pretty well. The model output is compared with the measured data in the left hand panel of Fig.8; the residuals are serially uncorrelated and the amplitude distribution of the normalized SDP error distribution appears reasonably Gaussian (see the right hand panel in Fig.8). Similar results are obtained with the IDIM-LS method but they are not shown here. In addition, the estimates of inertia, gravity and friction parameters are close to the reference values and the relative error obtained with the SDP-based algorithm is $4.5 \%$, less than the $5.8 \%$ obtained using the IDIM-LS method.

Examination of the results shows that there is one small but interesting difference between the nature of the estimated offsets obtained by IDIM and SDP estimation. These differences can be explained by the implementation of the SDP algorithm, which attempts to identify a separate offset for each state-dependent nonlinearity, with one offset identified for the friction and another for the gravity. However, by adding these two identified offsets together, we obtain the value given in Table 4, which is very close to the IDIM-LS-identified value. In other words, the SDP algorithm has 
conveniently separated the parameter offset 2 into two offsets (one for the friction and one for the gravity).

Table 4 : IDIM-LS and IDIM-SDP estimates compared with the reference values

\begin{tabular}{|c|c|c|c|}
\hline Parameters & IDIM-LS estimates & SDP estimates & Reference values \\
\hline$Z_{2}$ & $1.56(1.44 \%)$ & $\mathrm{X}$ & 1.60 \\
\hline$F_{v 2}$ & $5.52(2.08 \%)$ & $5.52(1.99 \%)$ & 5.68 \\
\hline$F_{c 2}$ & $7.06(0.47 \%)$ & $7.05(0.42 \%)$ & 7.77 \\
\hline$M X_{2}$ & $2.84(0.16 \%)$ & $2.83(0.14 \%)$ & 2.80 \\
\hline$M Y_{2}$ & $0.026(39.18 \%)$ & $0.045(38.38 \%)$ & 0.0 \\
\hline offset $_{2}$ & $0.055(8.61 \%)$ & $0.062(8.54 \%)$ & 0.0 \\
\hline relative error & $5.8 \%$ & $4.5 \%$ & $\mathrm{X}$ \\
\hline
\end{tabular}
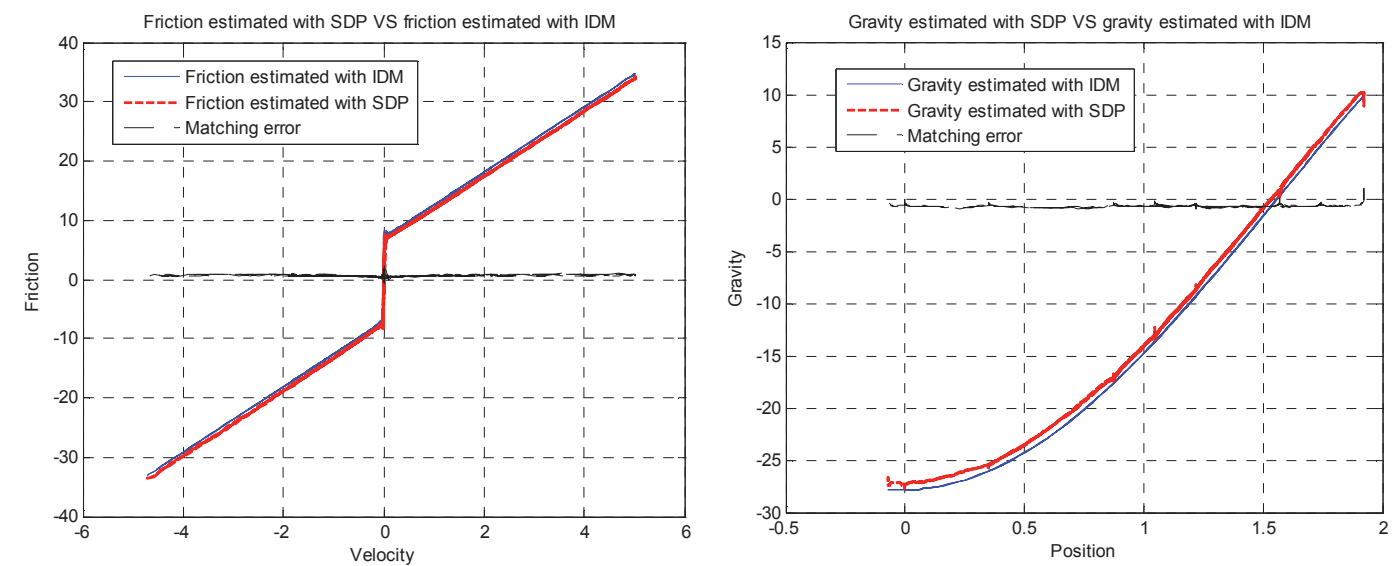

Fig.7.The Friction nonlinearity estimated by the non-parametric 1st stage SDP method compared with the friction effect identified with the IDIM-LS method (left panel); and a similar comparison between the two gravity effect nonlinearities in the right panel. 

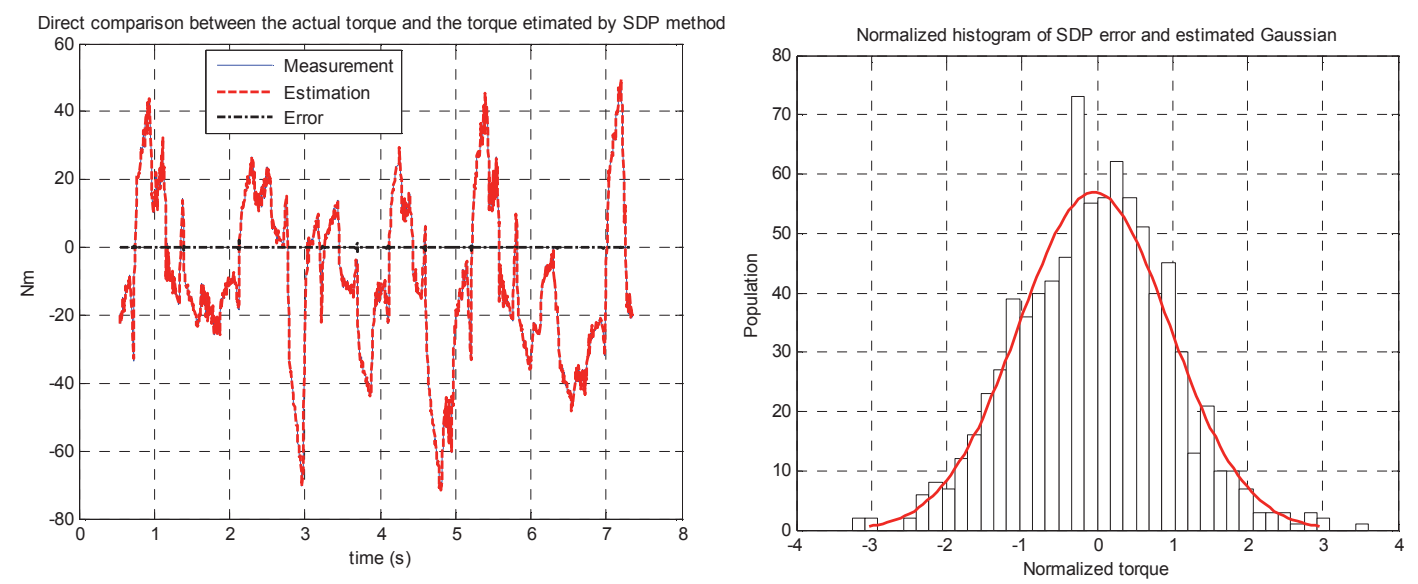

Fig.8. Direct comparison of the measured data with the output of the SDP-based identification method (IDIM-SDP, left panel) and histogram of the normalized error (right panel). A similar result is obtained with the IDIM-LS method.

\subsubsection{All the links are moving simultaneously}

In this situation, all the six joints of the TX40 robot are now excited with fifth-order polynomial trajectories that ensure good estimation of the dynamic characteristics. The dynamic parameters of the second link are again identified using SDP estimation. Here, the gear ratio is quite high i.e. greater than 10, so the second link is seen as a one-degree-of-freedom robot and its IDM is still given by (22). As in the previous examples, the SDP estimation results are similar to those obtained by the IDIM-LS method but they provide further insight into the detailed nature of the nonlinearity.

The IDIM-LS estimates are given in Table 5 and the comparison of the model output with the experimental data is shown in Fig.9. The estimates of inertia and friction parameters are quite close to the reference values but while the amplitude distribution of the normalized IDIM-LS error looks reasonably Gaussian, the error is serially correlated. Not surprisingly, therefore, the model output does not explain the measured data very well, with a relative error of $20.0 \%$. Such a result is a reason for concern because a relative error is expected to be less than $10 \%$.

As reported in section 3.4.1, the iterative SDP estimation procedure is initialized with $\hat{\mathbf{d}}_{q_{2}}^{0}\left(\hat{q}_{2}\right)$ and $\hat{\mathbf{d}}_{\dot{q}_{2}}^{0}\left(\hat{\dot{q}}_{2}\right)=\mathbf{0} ;$ tol $=1 \%$ is used as the convergence criterion; while the SDP nonlinearities $\hat{\mathbf{d}}_{q_{2}}^{k}\left(\hat{q}_{2}\right)$ and $\hat{\mathbf{d}}_{\dot{q}_{2}}^{k}\left(\hat{\dot{q}}_{2}\right)$ identified by this procedure are regressed on $\left[\begin{array}{lll}-g \cos \left(\hat{q}_{2}\right) & g \sin \left(\hat{q}_{2}\right) & 1\end{array}\right]$ and $\left[\begin{array}{ll}\hat{\dot{q}}_{2} & \operatorname{sign}\left(\hat{\dot{q}}_{2}\right)\end{array}\right]$, respectively. In this case, the algorithm converges in 6 iterations and the results plotted in Fig.10 demonstrate, together with the parameter estimates given in Table 5, that the gravity and friction shapes reconstructed by the algorithm do not match the gravity and the friction shapes reconstructed with the IDIM-LS estimates. The observed mismatches that can be seen in Fig.10 are due to the fact all the links are moving in the experiments and so some neglected coupling effects are being excited. Interestingly, the mismatches reflect, and so account for, such neglected coupling effects, so that the explanation of the data using the multi-SDP model, as shown in Fig.11, is rather better than that for the IDIM-LS estimated model in Fig.9. This confirmed by the calculation of the relative errors, $20.0 \%$ with the IDIM-LS method and $11.0 \%$ with the SDP method. 
These experimental results demonstrate once again the utility of the SDP estimation approach in highlighting where problems exist in nonlinear modelling and how they may be corrected. They also show how SDP estimation can be used as a tool in Data-Based Mechanistic (DBM) modelling. This is an inductive modelling strategy where less weight is placed on prior assumptions and more weight on the information in the experimental data. Only after carefully analysing the experimental data using appropriate model identification and signal processing tools, such as SDP estimation, does the modeller consider, at the mechanistic stage of the procedure, the prior assumptions and hypotheses, in order to see if these are compatible with the identified, data-based model. Or, if the data-based model is found to be deficient in any ways, as in this case when all the links are moving simultaneously, the modeller must consider whether new data need to be collected in order to examine these deficiencies using a better experimental design. And then, depending on the new SDP estimation results, the parametric form of the nonlinearities can be modified and re-estimated.

Table 5 : IDIM-LS and SDP estimates compared with the reference values

\begin{tabular}{|c|c|c|c|}
\hline Parameters & IDIM-LS estimates & SDP estimates & Reference values \\
\hline$Z_{2}$ & $0.9636(2.17 \%)$ & $\mathrm{X}$ & 1.09 \\
\hline$F_{v 2}$ & $5.2358(2.64 \%)$ & $5.1711(1.85 \%)$ & 5.68 \\
\hline$F_{c 2}$ & $7.8059(4.17 \%)$ & $7.8245(2.78 \%)$ & 7.77 \\
\hline$M X_{2}$ & $0.5636(16.53 \%)$ & $0.3573(7.31 \%)$ & 2.21 \\
\hline$M Y_{2}$ & $-3.6043(3.08 \%)$ & $-3.2864(1.07 \%)$ & 0.0 \\
\hline offset $_{2}$ & $12.8879(8.61 \%)$ & $9.5048(3.48 \%)$ & 0.0 \\
\hline relative error & $20.0 \%$ & $11.0 \%$ & $\mathrm{X}$ \\
\hline
\end{tabular}
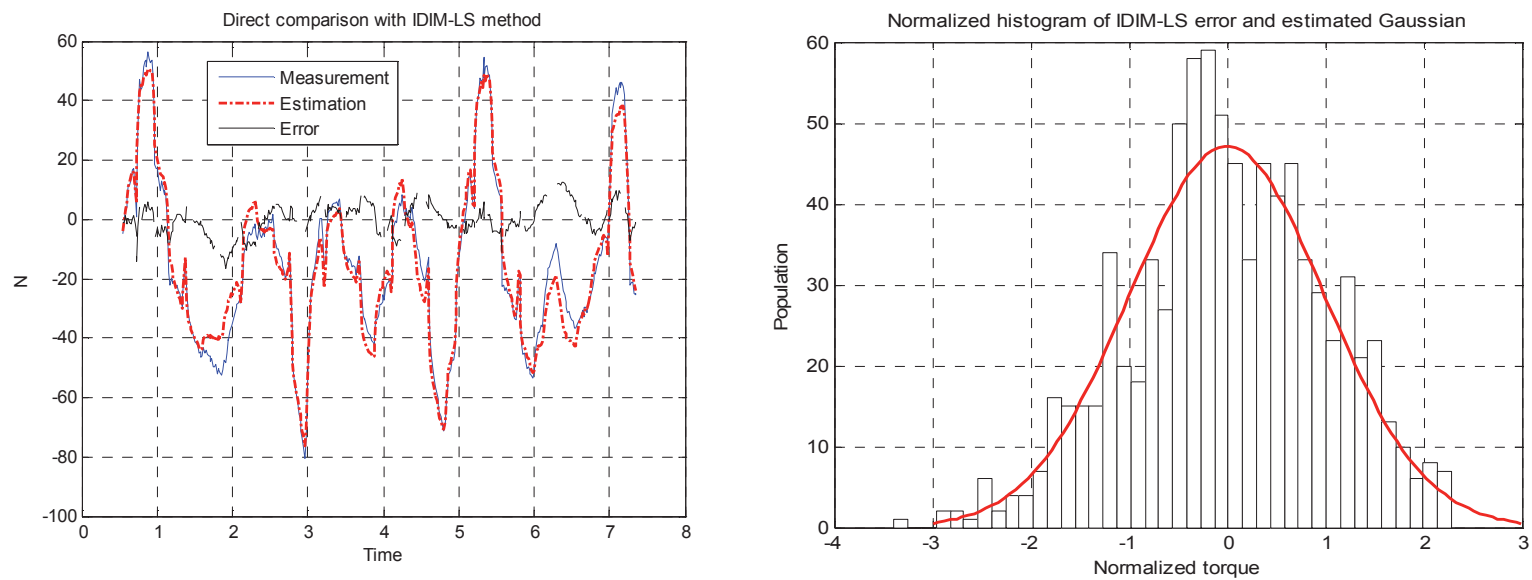

Fig.9. Direct comparison with the IDIM-LS method (left panel) and histogram of the normalized IDIM-LS error (right panel). The reconstructed torque does not match very well the measured one and although the error distribution is reasonably a Gaussian distribution the errors are serially correlated. This tends to show that the IDM given by $(23)$ is not well specified while all the joints are moving. 

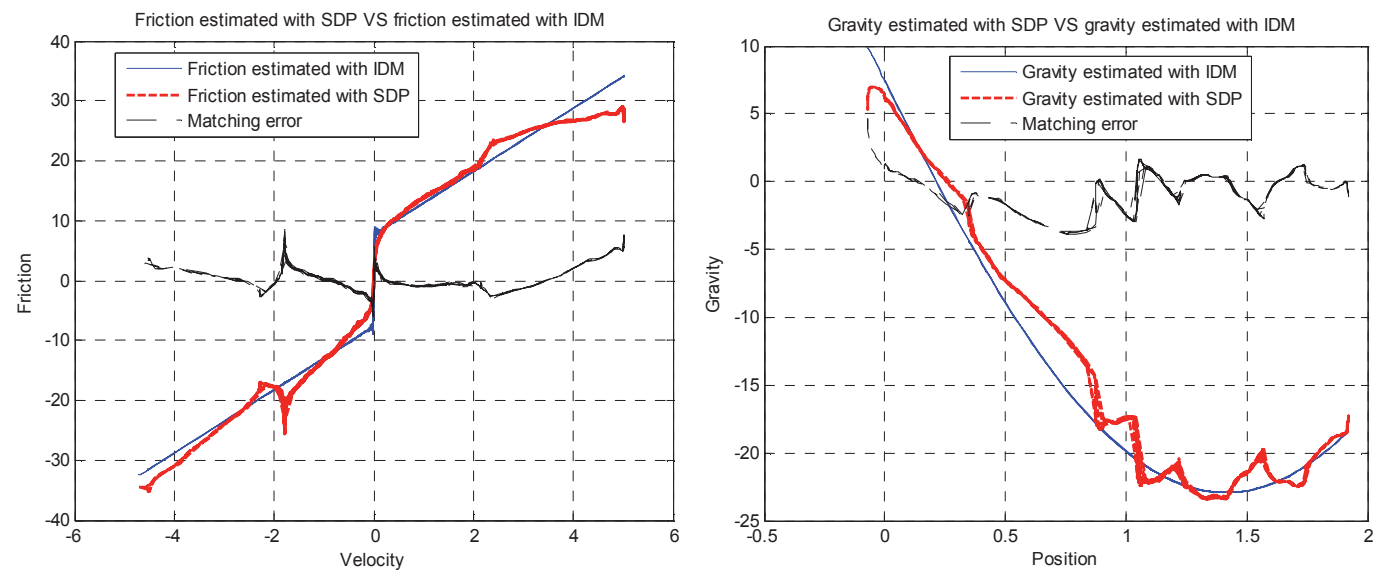

Fig.10: Friction shape reconstructed with the SDP method compared with the friction effect identified with the IDIM-LS method (left panel) and gravity shape reconstructed with the SDP method compared with the gravity effect identified with the IDIM-LS method (right panel). The mismatches observed suggest there are missing couplings.
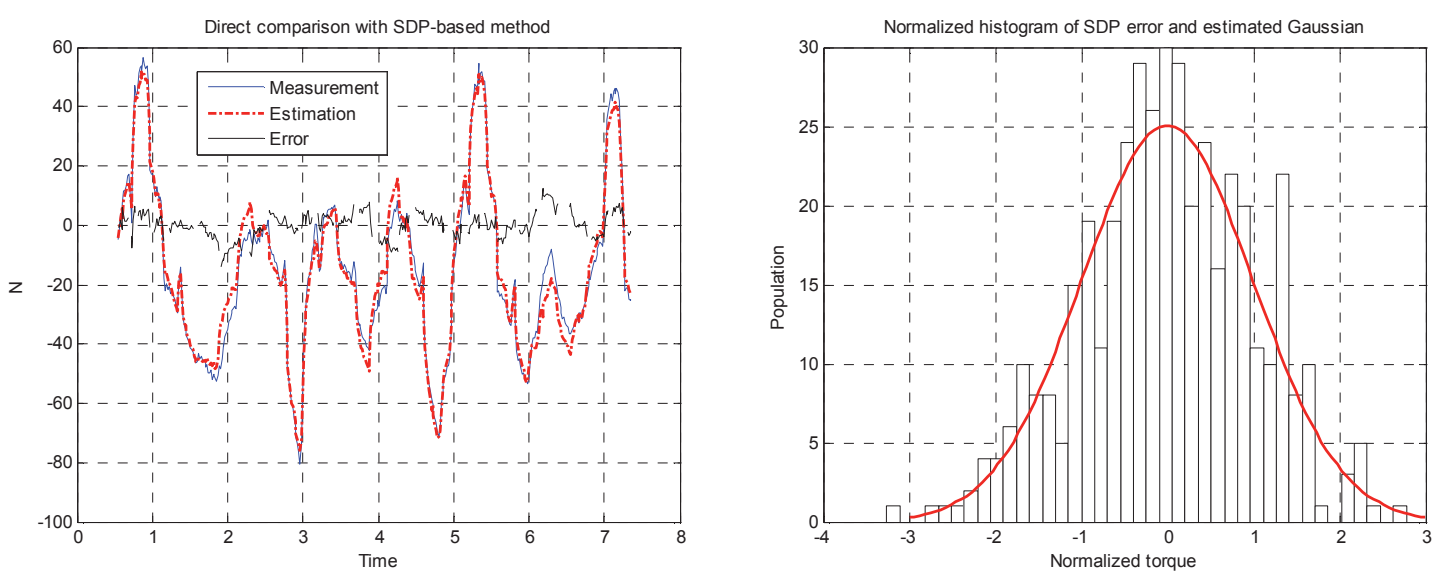

Fig.11. Direct comparison with the SDP-based method (left panel) and histogram of the normalized SDP-based error (right panel). A better matching between the reconstructed torque and the measured one is obtained. Furthermore, the error distribution is reasonably Gaussian but again there is some serial correlation. This shows that the IDM given by (23) is not well specified while all the joints are moving. This IDM must be therefore rejected.

\section{State-Dependent Parameter Control of the EMPS System}

One advantage of SDP nonlinear models is that they can form the basis for control system design based on the use of linear control theory: see (Taylor et al., 2008; and chapter 9 in Taylor et al., 2013). This SDP approach has some similarities with other methods that have been proposed, such as exact linearisation by feedback (Isidori, 1995) (better known as the computed torque in robotics: see 
Khalil \& Dombre 2002), velocity-based linearization (Leith \& Leithead, 1998); and Linear Parameter Varying (LPV) based control design (see e.g. White et al., 2013).

In this section, we consider how this methodology can be applied to the control of the simulated EMPS system represented by the DDM in equation (1), written as:

$\ddot{q}=c_{1} \dot{q}+c_{2} \operatorname{sign}(\dot{q})+c_{3}+c_{4} \tau$,

where,

$c_{1}=-F_{v} / M ; c_{2}=-F_{c} / M ; c_{3}=-o f f s e t / M ;$ and $c_{4}=1 / M$.

Based on the LS estimates given in Table 1, the values of these parameters are:

$c_{1}=-2.1277 ; c_{2}=-0.2123 ; c_{3}=0.0336 ;$ and $c_{4}=0.0105$.

\subsection{Derivation of the SDP control model}

Considering $u=\tau$ as the input and $x=q$ as the output, this estimated model can be represented as follows in transfer function form:

$$
\begin{aligned}
& x=\frac{c_{4}}{p\left(p+a_{\text {sdp }}\right)} u, \\
& y=x+w_{q}
\end{aligned}
$$

where $p^{r}=d^{r} / d t^{r}$ is the derivative operator; $w_{q}$ represents the additive noise with a noise/signal ratio by standard deviation of 5\%; and $a_{s d p}$ is an SDP estimated by the SDP routine in CAPTAIN using data from the prior closed loop experiments on the EMPS unit when controlled by the linear PD controller.

As we have seen in previous sections, $a_{s d p}$ defines the nonlinear characteristics of the open loop system and although it is denoted here as a parameter and used as such in the SDP control system design, it is a complete nonlinear function. This is illustrated in the Simulink model of the open-loop system appearing at the left of Fig.12 with the SDP nonlinearity block shown expanded at the right of the Fig.12. The functional form of $a_{s d p}$ is shown as the red part of the curve in Fig.13, while the blue parts of the curve are extrapolations to the estimated linear parts of the curve. These extensions of the relationship are required to handle larger fluctuations in the velocity arising from the more rapid SDP controlled response of the closed loop system. Note how the extrapolations reveal the asymmetry of the estimated nonlinearity, as exposed by the SDP estimation.

It will be seem that Fig.13 is a combination of the non-parametric SDP estimate and the parametric extrapolations. This combined form was chosen here, rather than the fully parametric form in equation (17), because it demonstrates how SDP control can be implemented directly using the nonparametric estimates, the parametric estimate or a hybrid combination of both, as here. This can be particularly useful if the estimated nonlinearity is rather complex, such as those shown in Fig.10, which would be more difficult to parameterize by simple relationships. 

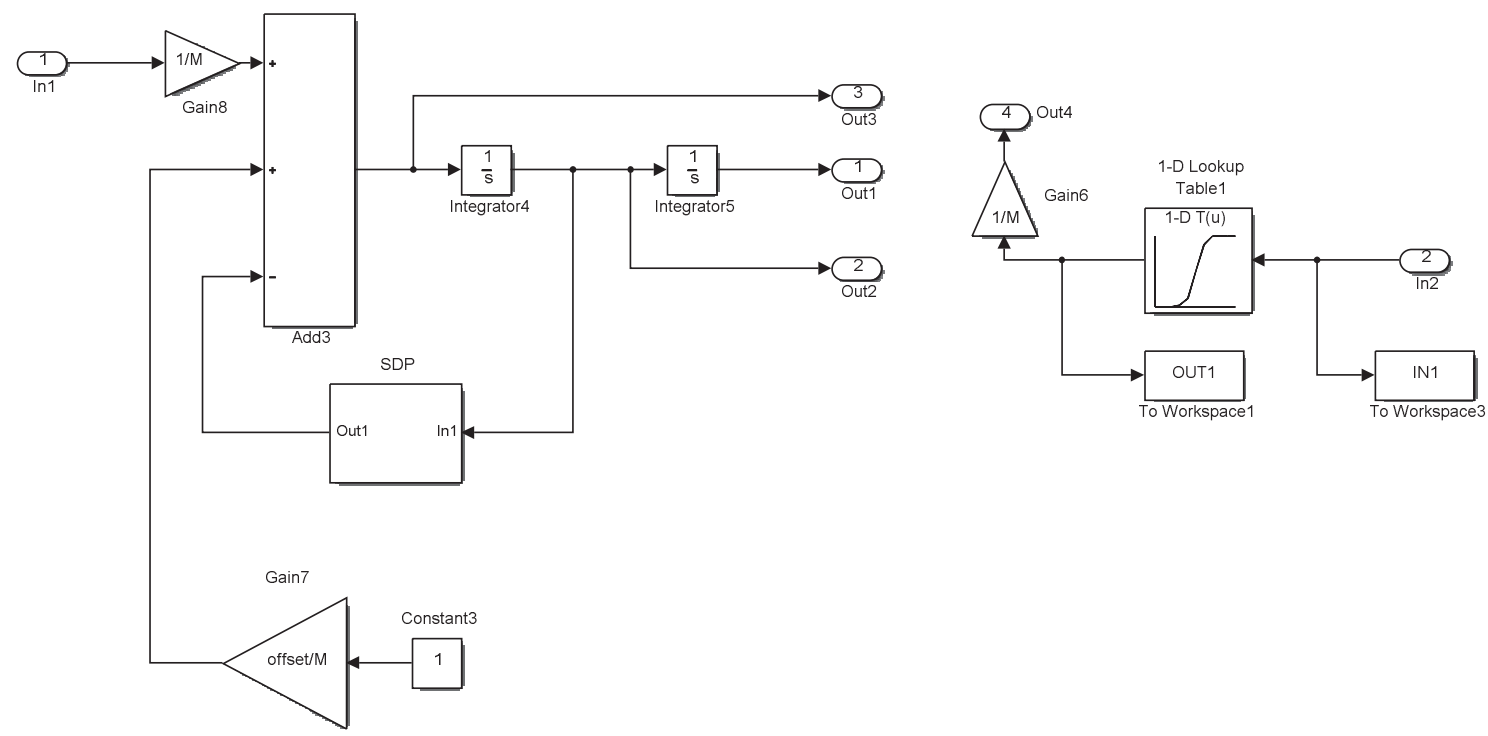

Fig.12. Simulation of the EMPS with the SDP-based control

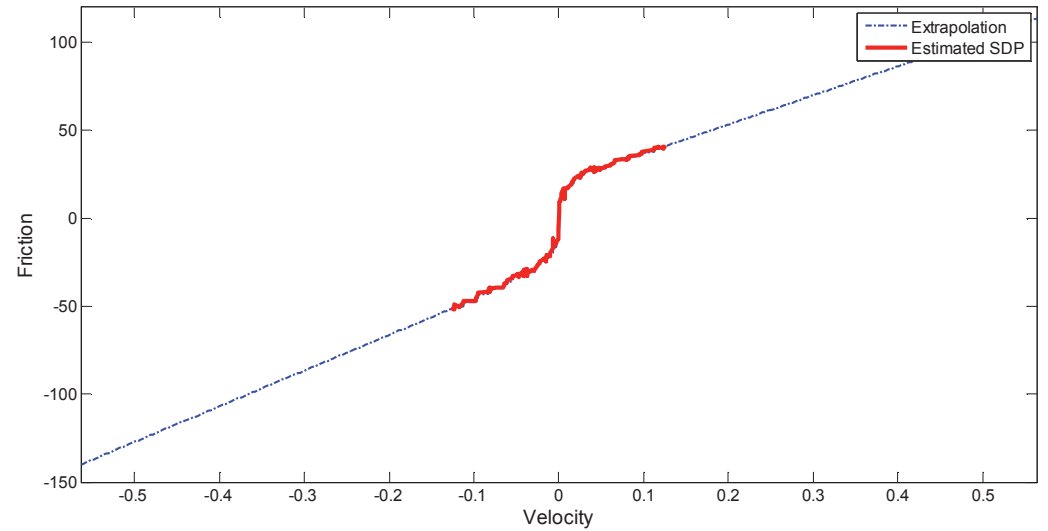

Fig.13. Friction model used with the SDP-based control

\subsection{SDP control system design: re-design of the PD controller}

The idea of using SDP models to simplify nonlinear control system design has a long heritage (see e.g. Young, 1981; Young, 1996). In the latter reference, it follows from research into linear control system design based on the Non-Minimal State Space (NMSS) form of the system model (see section 4.3). The NMSS control gains in the SDP case are effectively updated at each sampling instant, based on the linear "snapshot" of the SDP model at this sampling instant. Taylor et al. (2008) have shown that, using this approach, the stability of the closed loop non-linear system is guaranteed for "all-pole" systems, such as (27) and that good control system designs can be obtained for more general models. Although stability is not guaranteed in the case of model mismatch, the Monte Carlo-based uncertainty analysis reported in the paper suggests that the SDP/PIP approach is relatively robust to such uncertainty. 
As an initial exercise in SDP control system design, let us consider re-design of the simple PD controller used for the identification studies described in section 2.3 but based on the SDP transfer function model (27) and desired closed loop characteristics with $\eta=1.0 \quad$ (critical damping) and $\omega_{n}=250 \mathrm{rad} / \mathrm{s}$. As in section 2.3, the design is carried out by simple block diagram analysis with an SDP-based Proportional-Derivative (SDP-PD) pre-compensator $K_{p}+K_{v} p$. The closed loop transfer function obtained in this manner is given by

$$
x=\frac{c_{4}\left(K_{p}+K_{v} p\right)}{p^{2}+\left(a_{s d p}+c_{4} K_{v}\right) p+c_{4} K_{p}} r
$$

where $r$ is the command input, i.e. the reference. Since the system is second order, this PD control is equivalent to state variable feedback, so we see that both poles are assignable. And because the open-loop system model has a free integrator, the closed loop system exhibits "type 1" performance with unity gain and zero steady-state error to step command inputs.

If the desired closed loop TF denominator has damping $\eta_{d}$ and natural frequency $\omega_{n d}$, then we see that the values for the control gains can be computed from the equations:

$K_{p}=\omega_{n d}^{2} / c_{4} ; K_{v}=\left(2 \eta_{d} \omega_{n d}-a_{s d p}\right) / c_{4}$,

where it will be noted that the $K_{v}$ gain is a function of the SDP parameter $a_{s d p}$ and so the closed loop system synthesized with these gains includes the SDP nonlinearity, reflecting the nonlinear nature of the SDP-PD control system. In particular, because it is a state-dependent parameter, it changes or "adapts" in response to the changes in velocity.

The simulated response of the closed loop system to a step input command starting at zero, with a final value of $0.05 \mathrm{~m}$, is plotted in Fig.14 where it will be noted that the rapid response has a total settling time of 0.04 seconds. Also plotted in Fig.14 is the response of the conventional, linear PD controlled system used in the identification studies, where we see that the response is clearly much slower and oscillatory, with a total settling time about three times as long.

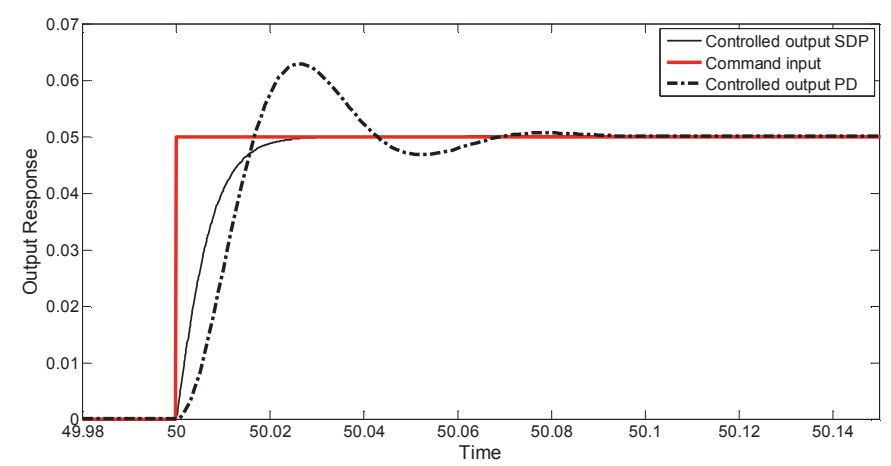

Fig.14. Output step response obtained with the SDP-based control (black solid line) and the PD control (black dash-dot line) 


\subsection{SDP control system design: PIP-SDP outer-loop control system design}

The NMSS-based Proportional-Integral-Plus (PIP) approach to control system design has been described comprehensively in the recent book by (Taylor et al., 2013), which includes all aspects of the design process, as well as numerous examples illustrating its application. In the present EMPS example, it could be applied directly, using the EMPS model in discrete-time NMSS form, but is would then require nonlinear modification of the kind described in previous sections, which is not straightforward once the model is transformed into discrete-time, digital form.

A simple, alternative approach is to implement PIP control as an "outer-loop" or "trimming" control that considers the SDP-PD controlled system, which is effectively linearized by its SDP mechanization, as the system to be controlled. The discrete-time model required for this design is obtained by statistical identification and estimation based on input-output data from the SDP-PD controlled system using optimal Refined Instrumental Variable (RIV) estimation for continuous time systems (see Young, 2011), as implemented by the RIVCBJID and RIVCBJ routines in the CAPTAIN Toolbox.

The discrete-time model required for PIP control system design is then obtained via the Matlab c2d continuous to discrete-time conversion routine. The sampling interval for such conversion is at the discretion of the control system designer but, in this case, the discrete-time model so obtained, for a sampling interval of $0.01 \mathrm{~s}$, is:

$x(k)=\frac{0.0107 z^{-1}-0.0088 z^{-2}+0.0025 z^{-1}}{1-1.8660 z^{-1}+0.8706 z^{-2}} r(k)$,

where $z^{-r}$ is the backward shift operator, i.e. $z^{-r} x(k)=x(k-r)$.

The PIP design for this model system is based on Linear-Quadratic (LQ) optimization of the associated NMSS model form, where the NMSS control gains are computed by the the PIPOPT and GAINS routines in the CAPTAIN Toolbox. These use the numerator and denominator model coefficients in (30) together with the user specified weightings on the error, ew; control input, $u w$; and the nonminimal state variables $x w$ where, in this example, $e w=10 ; u w=1.0$; and $x w=1.0$. A serially connected loop gain $L G=5.0$ is added to the design in order to tune the closed loop response so that it just meets the constraint imposed by a required $0.15 \mathrm{~m} / \mathrm{s}$ velocity limit.

The full details of this PIP-SDP control system design and evaluation are given in (Young, 2015). This shows that the closed loop system responds well to any violation of hard constraints and is not sensitive to uncertainty in the estimated model parameters, including the SDP nonlinearity, unless these reach very high levels. Consequently, this control system design represents a reasonable, simulation-based starting point for future planned research and development studies.

\section{Conclusions}

This paper has shown how the concept of State-Dependent Parameter (SDP) models for nonlinear dynamic systems can be exploited to aid the identification and control of electro-mechanical systems. It has demonstrated how SDP identification provides an alternative to the existing standard 
methods of statistical identification for such systems; an alternative that can help to avoid overreliance on prior conceptions about the nature of the nonlinear characteristics.

When used as a tool in the experimental evaluation of an Electro-Mechanical Positioning System (EMPS), the first, non-parametric estimation stage in the SDP identification procedure is able to discover deviations from the assumed nonlinear characteristics of the system and quantify the resulting nonlinear characteristics in a practically useful SDP form. The second IDIM-SDP stage, based on least squares estimation of the suitably parameterized SDP model, can be considered as a logical improvement of the standard IDIM-LS method. One application of such SDP models is to facilitate nonlinear control system design using linear-like design procedures. This is illustrated by simulation studies that show how the SDP model of the EMPS system can be used as the basis for the SDP Proportional-Integral-Plus (SDP-PIP) design of a nonlinear control system for the EMPS.

SDP identification is one of the tools used for the Data-Based Mechanistic (DBM) modelling of dynamic systems. This general, inductive method of modelling differs from the alternative, hypothetico-deductive "grey-box" approach that is often used for identifying electro-mechanical systems. In particular, only after initial, purely data-based "black-box" modelling are any prior assumptions and hypotheses considered in order to see if they are compatible with the identified model, or whether new data need to be collected in order to examine any significant differences. A typical example of how SDP identification can be exploited in such a diagnostic role is demonstrated by the results of experiments that show SDP identified deficiencies in the initially assumed nonlinear characteristics of the Stäubli TX40 robot system.

\section{References}

Beven, K. J., Leedal, D. T., Smith, P. J., and Young, P. C., 2012, Identification and representation of state dependent nonlinearities in flood forecasting using the DBM methodology. System Identification, Environmetric Modelling and Control (London: Springer-Verlag), pp 341-366.

Brunot, M., Janot, A., Carrillo, F., Garnier, H., Vandanjon, P.O., and Gautier, M., 2015. Physical parameter identification of a one-degree-of-freedom electromechanical system operating in closed loop. Proceedings of the17th IFAC Symposium on System Identification (SYSID 2015), Beijing, China.

Calanca, A., Capisani, L. M., Ferrara, A., and Magnani, L., 2011, MIMO Closed Loop Identification of an Industrial Robot. IEEE Transactions on Control System Technology, 19, 1214-1224.

Gautier ,M., and Khalil, W., 1992, Exciting trajectories for the identification of the inertial parameters of robots. International Journal of Robotics Research, 11, 362-375.

Gautier, M., and Poignet, P., 2001, Extended Kalman Filtering and Weighted Least-squares Dynamic Identification of Robot. Control Engineering Practice, 9, 1361-1372.

Gautier, M., Janot, A., and Vandanjon, P.O., 2013, A New Closed-Loop Output Error Method for Parameter Identification of Robot Dynamics. IEEE Transactions on Control System Technology, 21, 428-444.

Gautier, M., and Briot, S., 2014, Global Identification of Joint Drive Gains and Dynamic Parameters of Robots. Journal of Dynamic Systems, Measurement, and Control, 136, 1-9. 
Hollerbach, J., Khalil, W., and Gautier, M., 2008, Springer Handbook of Robotics (Berlin Heidelberg: Springer).

Indri, M., Calafiore, G., Legnani, G., Jatta, F., and Visioli A., 2002, Optimized Dynamic Calibration of a SCARA Robot. Proceedings of the 15th IFAC World Congress (IFAC WC 2002), Barcelona, Spain.

Isidori, A., 1995, Nonlinear Control Systems (Berlin: Springer-Verlag).

Janot, A., Vandanjon, P.O., and Gautier, M., 2014a.A Generic Instrumental Variable Approach for Industrial Robots Identification. IEEE Transactions on Control Systems Technology, 22, 132-145.

Janot, A., Vandanjon, P.O., and Gautier, M., 2014b, An instrumental variable approach for rigid industrial robots identification. Control Engineering Practice, 25, 85-101.

Khalil, W., and Dombre, E., 2002, Modeling, identification and control of robots (London: Hermes Penton).

Kostic, D., de Jager, B., Steinbuch, M., and Hensen, R., 2004, Modeling and Identification for HighPerformance Robot Control: An RRR-Robotic Arm Case Study. IEEE Transactions on Control System Technology, 12, 904-919.

Leith, D. J., and W. E., Leithead, 1998, Gain-scheduled and nonlinear systems: Dynamic analysis by velocity-based linearization families. International Journal of Control, 70, 289-317.

Noel, J-P., Schoukens, J., and Kerschen, G., 2015, Grey-box nonlinear state-space modelling for mechanical vibrations identification. Proceedings of the 17th IFAC Symposium on System Identification (SYSID 2015), Beijing, China.

Olsen, M. M., Swevers, J., and Verdonck, W., 2002, Maximum Likelihood Identification of a Dynamic Robot Model: Implementation Issues. International Journal of Robotics Research, 21, 89- 96.

Östring, M., Gunnarsson, S., Norrlöf, M., 2003, Closed-loop identification of an industrial robot containing flexibilities. Control Engineering Practice, 11, 291-300.

Previdi, F., and Lovera, M., 2003, Identification of a class of nonlinear parametrically varying models. International Journal on Adaptive Control and Signal Processing, 17, 33-50.

Previdi, F.,Lovera, M., Identification of non-linear parametrically varying models using separable least squares. International Journal of Control, 77, 1382-1392.

Ramdani, N., and Poignet, P., 2005, Robust Dynamic Experimental Identification of Robots with Set Membership Uncertainty. IEEE/ASME Transactions on Mechatronics, 10, 253 - 256.

Sadeghi, J., Tych, W., Chotai, A., and Young, P.C., 2010, Multi-state dependent parameter model identification and estimation for nonlinear dynamic systems. Electronics Letters, 46, 1265 - 1266.

Swevers, J., Verdonck, W., and De Schutter, J., 2007, Dynamic model identification for industrial robots - Integrated experiment design and parameter estimation. IEEE Control Systems Magazine, 27, 58-71. 
Taylor, C. J., A. Chotai, and P. C., Young, 2008, Non-linear control by input-output state variable feedback pole assignment. International Journal of Control, 82, 1029-1044.

Taylor, C. J., P. C., Young, and A., Chotai, 2013, True Digital Control. Chichester: Wiley.

Van den Hof, P.M.J., 1998, Closed loop issues in system identification. Annual Reviews in Control, 22, 173-186.

Wernholt, E., and Gunnarsson, S., 2008, Estimation of Nonlinear Effects in Frequency Domain Identification of Industrial Robots. IEEE Transactions on Instrumentation and Measurement, 57, 856863.

White, A. P., Zhu, G., and Choi, J., 2013, Linear Parameter-Varying Control for Engineering Applications (London: Springer-Verlag).

Xi, F., 1995, Effect of Non-Geometric Errors on Manipulator Inertial Calibration, Proceedings of International Conference on Robotics and Automation (ICRA 1995), Nagoya, Japan.

Young, P.C., 1981, A second generation adaptive autostabilization system for airborne vehicles. Automatica, 17, 459-470.

Young, P.C., 1996, A general approach to identification, estimation and control for a class of nonlinear dynamic systems, in M. I. Friswell and J. E. Mottershead (Eds.), Identification in Engineering Systems, University of Wales: Swansea.

Young, P.C., 1998, Data-based mechanistic modeling of engineering systems. Journal of Vibration and Control, 4, pp. 5-28.

Young, P.C., 1998, Data-based mechanistic modeling of environmental, ecological, economic and engineering systems. Environmental Modelling \& Software, 13, 105-122,.

Young, P.C., 1999, Nonstationary time series analysis and forecasting. Progress in Environmental Science, 1, 3-48, 1999.

Young, P.C., 2000, Stochastic, dynamic modelling and signal processing: time variable and state dependent parameter estimation, in Nonlinear and Nonstationary Signal Processing, W.J. Fitzgerald, A. Walden, R. Smith and P.C. Young, Eds, Cambridge: Cambridge University Press, 2000, pp. 74-114.

Young. P. C. , 2001, The identification and estimation of nonlinear stochastic systems. in A. I. Mees, editor, Nonlinear Dynamics and Statistics (Birkhauser: Boston), pp 127-166.

Young, P.C., 2005, Comments on Identification of non-linear parametrically varying models using separable least squares' by F. Previdi and M. Lovera: black-box or open box? International Journal of Control, 78, 122-127.

Young, P.C., 2011, Recursive Estimation and Time-Series Analysis: An Introduction for the Student and Practitioner (Berlin: Springer-Verlag). 
Young, P.C., 2015, SDP control of second order electro-mechanical positioning systems. Technical Report TN/PCY/2, Systems and Control Group, Lancaster Environment Centre, Lancaster University, UK.

Young, P.C., Foster, M., and Lees, M. J., 1993, A direct approach to the identification and estimation of continuous-time systems from discrete-time data based on fixed interval smoothing. Proceedings 12th IFAC World Congress (IFAC WC 1993), Sydney, Australia.

Young, P.C., McKenna, P., and Bruun, J., 2001, Identification of nonlinear stochastic systems by state dependent parameter estimation. International Journal of Control, 74, 1837-1857. 